\title{
General magnetized Weyl solutions: Disks and motion of charged particles
}

\author{
Cristian H. García-Duque* and Gonzalo García-Reyesđ \\ Universidad Tecnológica de Pereira, Departamento de Física, A. A. 97, Pereira, Colombia
}

\begin{abstract}
We construct three families of general magnetostatic axisymmetric exact solutions of EinsteinMaxwell equations in spherical coordinates, prolate, and oblates. The solutions obtained are then presented in the system of generalized spheroidal coordinates which is a generalization of the previous systems. The method used to build such solutions is the well-known complex potential formalism proposed by Ernst, using as seed solutions vacuum solutions of the Einstein field equations. We show explicitly some particular solutions among them a magnetized Erez-Rosen solution and a magnetized Morgan-Morgan solution, which we interpret as the exterior gravitational field of a finite dislike source immersed in a magnetic field. From them we also construct using the well known "displace, cut and reflect" method exact solutions representing relativistic thin disks of infinite extension. We then analyze the motion of electrically charged test particles around these fields for equatorial circular orbits and we discuss their stability against radial perturbations. For magnetized MorganMorgan fields we find that inside of disk the presence of magnetic field provides the possibility of to find relativist charged particles moving in both prograde and retrograde direction.
\end{abstract}

\footnotetext{
* e-mail: garciahcristian@hotmail.com

$\dagger$ e-mail: ggarcia@utp.edu.co
} 


\section{INTRODUCTION}

Magnetic fields play an important role in the study of astrophysical objects such as neutron stars, white dwarfs, pulsars, black holes and galaxy formation. In fact, several observations show that there are various scenarios where the magnetic fields and general relativity can not be neglected. One of them is the presence of strong magnetic fields in active galactic nuclei 1 - 4]. These nuclei are known to produce more radiation than the rest of the entire galaxy and directly affect its structure and evolution. Another scenario is the production of relativistic collimated jets in the inner regions of accretion discs, which can be explained considering magneto-centrifugal mechanisms [5-10]. Also, magnetic fields are important in understanding the interplay between magnetic and thermal processes for strongly magnetic neutron stars $[11-13]$. At least $10 \%$ of all neutron stars are born as magnetars, with magnetic fields above $10^{14} \mathrm{G}$ [14 16]. Analytical models that describe these astrophysical objects are often associated with solutions of Einsteins equations 17 22]. In the search for more realistic models for compact stellar systems, the energy-momentum tensor, the source of Einsteins equations, is modified by introducing more complex terms that take into account additional physical properties as, for example, electromagnetic fields [23].

Stationary or static axially symmetric exact solutions to the Einstein field equations representing relativistic thin disks are of great astrophysical importance since they can be used as models for certain galaxies, accretion disks, and the superposition of a black holes and a galaxy or an accretion disk as in the case of quasars. Static thin disks without radial pressure were first studied by Bonnor and Sackfield [88], and Morgan and Morgan [25], and with radial pressure by Morgan and Morgan [26]. Also thin disks with radial tension were considered [27]. Several classes of exact solutions of the Einstein field equations corresponding to static thin disks with or without radial pressure have been obtained by different authors [28, 30 35]. Rotating thin disks that can be considered as a source of a Kerr metric were presented by Bičák and Ledvinka [36], while rotating disks with heat flow were were studied by González and Letelier [37]. Disk sources for stationary axially symmetric spacetimes with electromagnetic fields, especially magnetic fields, are also of astrophysical importance in the study of neutron stars, white dwarfs and galaxy formation. In such situation one has to study the coupled Einstein-Maxwell equations. Thin disks in presence of electromagnetic field have been discussed as sources for Kerr-Newman fields [38, 39], magnetostatic axisymmetric fields [40], conformastationary metrics [41], while models of electrovacuum static counterrotating dust disks were presented in [42]. Charged perfect fluid disks were also studied by Vogt and Letelier [43], and charged perfect fluid disks as sources of static and Taub-NUT-type spacetimes by García-Reyes and González [44, 45].

In all the above cases, the disks are obtained by an "inverse problem" approach, called by Synge the " $g$-method" [46]. The method works as follows: a solution of the vacuum Einstein equations is taken, such that there is a discontinuity in the derivatives of the metric tensor on the plane of the disk, and the energy-momentum tensor is obtained from the Einstein equations. The physical properties of the matter distribution are then studied by an analysis of the surface energy-momentum tensor so obtained. Another approach to generate disks is by solving the Einstein equations given a source (energy-momentum tensor). Essentially, they are obtained by solving a Riemann-Hilbert problem and are highly nontrivial [47 53]. A review of this kind of disks solutions to the Einstein-Maxwell equations was presented by Klein in [54].

Motion of matter near compact stars and black holes has been discussed widely in literature. The interplay between gravitational and electromagnetic interaction is essential for characteristics of the motion, namely its stability properties. Motivation for these studies arises from the problem of motion and acceleration of matter (charged particles or dust grains) [55 58]. The study of the interaction between particles and electromagnetic fields in curved spacetimes is also of astrophysical interest, such is the case of strong synchrotron radiation emerging galactic cores, which can be explained admitting the existence in those regions of extended and very intense magnetic fields, interacting with ultrarelativistic electrons. Such magnetic fields could originate in the inner part of an accretion disc around the central black hole [59, 60]. Also have been shown that the presence of a strong magnetic field provides the possibility of relativistic motion of counterrrotating matter [61].

In the present work we construct three families of general magnetostatic axisymmetric exact solutions of EinsteinMaxwell equations in spherical coordinates, prolate, and oblates. The zero order of this solutions were investigated previously in the Ref. [42], and although the models of thin disks constructed there satisfied all the energy conditions, the solutions are not asymptotically flat. Thus the solutions presented in this work are a generalization of solutions discussed in that reference, and, except the zero order, the new families of solutions are asymptotically flat but with total mass zero. Hence, all discussed solutions with magnetic field are either singular, not asymptotically flat or massless. Moreover, in all cases we build models of thin disk in which all the energy conditions are satisfied, which characterizes a matter distribution made of usual matter. In the case of oblates coordinates, the built solutions correspond to the magnetized version of the Morgan-Morgan vacuum solution [25]. These solutions are interpreted as the exterior gravitational field of a finite dislike matter distribution immersed in a magnetic field, and thus can be used to model disklike astrophysical objects such as galaxies, accretion disks, and certain stars in presence of magnetic fields. However, unlike the part material or the electrostatic case, we show that the source of the magnetic field is not 
planar but of a different origin such as a remnants or fossil magnetic field [62], or can come from external sources, such as the presence of a nearby magnetars or neutron stars. On the other hand, even though realistic disklike sources have thickness, in first approximation these astrophysical objects can be considered to be very thin, e.g., in our Galaxy the radius of the disk is $10 \mathrm{kpc}$ and its thickness is $1 \mathrm{kpc}$. In all cases we also construct relativistic models of infinite thin disks. We find for all the values of parameters that the surface energy density decreases rapidly which permits one can define a cut off radius and, in principle, to consider these disks as finite. In addition, we analyze the motion of charged test particles around these fields for circular equatorial orbits and we discuss the stability of this orbits against radial perturbations. For magnetized Morgan-Morgan fields we find that inside of disk the presence of magnetic field provides the possibility of to find relativist charged particles moving in both prograde and retrograde direction. Even though this result can be seen as merely theoretical, there are observational evidence of counterrotating matter components in certain types of galaxies $[6367]$.

The paper is organized as follows. In section II we make a review of the Ernst's method in the case of a magnetostatic axisymmetric spacetime and we construct three families of solutions of Einstein-Maxwell equations in spherical coordinate, prolate and oblate, and then in generalized spheroidal coordinates which are a generalization of the previous cases. We also show explicitly some particular solutions among them a magnetized Erez-Rosen solution and magnetized Morgan-Morgan solution, and we analyze limiting cases.

In section III we present a summary of the procedure to obtain models of thin disks with a purely azimuthal pressure and currents for the Einstein-Maxwell equations. In particular, we obtain expressions for the surface energymomentum tensor and the surface current density of the disks. In the case of the magnetized Morgan-Morgan solutions, these are interpreted as the exterior gravitational field of a finite dislike source immersed in a magnetic field. Then we construct using the well known "displace, cut and reflect" method exact solutions representing relativistic thin disks of infinite extension.

In section IV we study for some particular solutions the equatorial circular motion of charged test particles and we also discuss their stability against radial perturbation using an extension of Rayleigh criteria of stability [68 70]. In particular we analyze the circular velocity and the specific angular momentum of the particles. In order to compare the behavior of these physical quantities with other known magnetized solutions, we also study the electrogeodesic motion of test particles and their stability for a Kerr-type solution or magnetic dipole solution [74]. Finally, in Section $\mathrm{V}$ we summarize and discuss the results obtained.

\section{GENERAL MAGNETIZED WEYL SOLUTIONS}

The simplest metric to describe a static axially symmetric spacetime is the Weyl's line element 73 ]

$$
d s^{2}=-e^{2 \psi} d t^{2}+e^{-2 \psi}\left[\rho^{2} d \varphi^{2}+e^{2 \Lambda}\left(d \rho^{2}+d z^{2}\right)\right],
$$

where $(t, \varphi, \rho, z)$ are the Weyl canonical coordinates, and $\psi$ and $\Lambda$ are functions of the coordinates $\rho$ and $z$ only. For the coordinates we also use the notation $\left(x^{0}, x^{1}, x^{2}, x^{3}\right)=(t, \varphi, \rho, z)$. The vacuum Einstein-Maxwell equations, in geometrized units such that $G=c=1$, are given by

$$
\begin{aligned}
R_{a b} & =8 \pi T_{a b}, \\
T_{a b} & =\frac{1}{4 \pi}\left[F_{a c} F_{b}{ }^{c}-\frac{1}{4} g_{a b} F_{c d} F^{c d}\right], \\
F_{; b}^{a b} & =0, \\
F_{a b} & =A_{b, a}-A_{a, b},
\end{aligned}
$$

where $T_{a b}$ is the electromagnetic energy-momentum tensor, $F_{a b}$ is the electromagnetic field tensor, and $A_{a}=(\phi, A, 0,0)$ is the four-potential, where $\phi$ is the electric potential and $A$ the magnetic potential which are also functions of $r$ and $z$ only. Further, ()$_{a}=\partial / \partial x^{a}$, and ()$_{; a}$ means covariant derivate.

For the metric (1) and in magnetostatic case, the Einstein-Maxwell equations are

$$
\begin{aligned}
\nabla \cdot\left[\rho^{-2} f \nabla A\right] & =0, \\
f \nabla^{2} f & =\nabla f \cdot \nabla f+2 \rho^{-2} f^{3} \nabla A \cdot \nabla A, \\
\Lambda,,_{\rho} & =\rho\left(\psi^{2},,_{\rho}-\psi^{2},{ }_{z}\right)+\frac{1}{\rho}\left(A^{2},,_{\rho}-A^{2},{ }_{z}\right) f, \\
\Lambda,,_{z} & =2 \rho \psi,,_{\rho} \psi,_{z}+\frac{2}{\rho} A,_{\rho} A,_{z} f,
\end{aligned}
$$


where $f=e^{2 \psi}$. The equations (3a) y (3b) are equivalent to [71, 72]

$$
\begin{aligned}
& f \Delta \mathcal{E}=\left(\nabla \mathcal{E}+2 \Phi^{*} \nabla \Phi\right) \cdot \nabla \mathcal{E}, \\
& f \Delta \Phi=\left(\nabla \mathcal{E}+2 \Phi^{*} \nabla \Phi\right) \cdot \nabla \Phi,
\end{aligned}
$$

where $\Delta$ and $\nabla$ are the standard differential operators in cylindrical coordinates, $f=e^{2 \psi}$, and $\mathcal{E}$ and $\Phi$ are complex potentials which in the case static $\mathcal{E}=\mathcal{E}^{*}$ and for the case magnetostatic $\Phi^{*}=-\Phi$. The above equations are called Ernst equations. The metric functions are obtained via

$$
\begin{aligned}
f & =\mathcal{E}+\Phi \Phi^{*}, \\
\Lambda_{, \zeta} & =\frac{\sqrt{2} \rho}{4 f^{2}}\left(\mathcal{E}_{, \zeta}+2 \Phi^{*} \Phi_{, \zeta}\right)\left(\mathcal{E}_{, \zeta}+2 \Phi \Phi_{, \zeta}^{*}\right)-\frac{\sqrt{2} \rho}{f} \Phi_{, \zeta} \Phi_{, \zeta}^{*},
\end{aligned}
$$

where $\sqrt{2} \zeta=\rho+i z$, so that $\sqrt{2} \partial_{, \zeta}=\partial_{, \rho}-i \partial_{, z}$, and the magnetic potential $A$ is related to $\Phi$ via

$$
A_{, \zeta}=i \frac{\rho}{f}(\operatorname{Im} \Phi)_{, \zeta}
$$

Taking $\mathcal{E}$ as function of $\Phi$, from (4a) and (4b) it follows that

$$
\left(\operatorname{Re} \mathcal{E}+|\Phi|^{2}\right) \frac{d^{2} \mathcal{E}}{d \Phi^{2}} \nabla \Phi \cdot \nabla \Phi=0,
$$

and hence that $\mathcal{E}$ is a lineal function of $\Phi$. Using the boundary conditions $\mathcal{E} \rightarrow 1$ and $\Phi \rightarrow 0$ at infinity, we obtain

$$
\mathcal{E}=1-2 q^{-1} \Phi
$$

where $q$ is a complex constant. With the change of variable

$$
\mathcal{E}=\frac{\xi-1}{\xi+1}
$$

then (8) implies

$$
\Phi=\frac{q}{(\xi+1)}
$$

and the Ernst equations read

$$
\left[\xi \xi^{*}-\left(1-q q^{*}\right)\right] \nabla^{2} \xi=2 \xi^{*} \nabla \xi \cdot \nabla \xi
$$

Then making $\xi=\left(1-q q^{*}\right)^{1 / 2} \hat{\xi}$ the equation (11) takes of form

$$
\left(\hat{\xi} \hat{\xi}^{*}-1\right) \nabla^{2} \hat{\xi}=2 \hat{\xi}^{*} \nabla \hat{\xi} \cdot \nabla \hat{\xi}
$$

which is the Ernst equation in the vacuum [72]. So given a solution of the Einstein field equations in vacuum $\hat{\xi}$ (seed solution) we can construct a solution of the Einstein-Maxwell field equations. A solutions for this equations is

$$
\hat{\xi}=-e^{i \alpha} \operatorname{coth} \hat{\psi}
$$

where the function $\hat{\psi}$ satisfies the Laplace's equation

$$
\nabla^{2} \hat{\psi}=0
$$

The case $\alpha=0$ corresponds to the well-known Weyl vacuum solutions and the magnetized solutions (taking $\Phi$ imaginary) built from them are the magnetized Weyl solutions. The metric functions and magnetic potential are given by

$$
\begin{aligned}
f & =\frac{4}{\left[(1+a) e^{-\hat{\psi}}+(1-a) e^{\hat{\psi}}\right]^{2}}, \\
\Lambda & =\hat{\Lambda} \\
A_{, \rho} & =-b \rho \hat{\psi}_{, z}, \\
A_{, z} & =b \rho \hat{\psi}_{, \rho},
\end{aligned}
$$


where $a=\sqrt{1+b^{2}}$, being $b$ is the parameter that controls the magnetic field, and $\hat{\Lambda}$ is the metric potential $\Lambda$ corresponding to the seed solution, that is taking $\mathcal{E}=e^{2 \hat{\psi}}$ and $\Phi=0$.

In terms of generalized spheroidal coordinates $(\xi, \eta)[89]$ which are related to Weyl coordinates $(\rho, z)$ by

$$
\begin{aligned}
\rho^{2} & =k^{2}\left(\xi^{2}-\sigma^{2}\right)\left(1-\eta^{2}\right), \\
z & =k \xi \eta,
\end{aligned}
$$

with $k$ and $\sigma$ constants, the equations for the magnetic potential (15c) - (15d) can be cast as

$$
\begin{aligned}
& A_{, \xi}=-k b\left(1-\eta^{2}\right) \hat{\psi}_{,_{\eta}}, \\
& A_{,_{\eta}}=k b\left(\xi^{2}-\sigma^{2}\right) \hat{\psi},_{\xi} .
\end{aligned}
$$

and for the function $\Lambda$

$$
\begin{gathered}
\Lambda_{, \xi}=\left(\frac{1-\eta^{2}}{\xi^{2}-\sigma^{2} \eta^{2}}\right)\left[\xi\left(\xi^{2}-\sigma^{2}\right) \hat{\psi}_{, \xi}^{2}-\xi\left(1-\eta^{2}\right) \hat{\psi}_{,_{\eta}}^{2}-2 \eta\left(\xi^{2}-\sigma^{2}\right) \hat{\psi},_{\xi} \hat{\psi}_{, \eta}\right] \\
\Lambda_{, \eta}=\left(\frac{\xi^{2}-\sigma^{2}}{\xi^{2}-\sigma^{2} \eta^{2}}\right)\left[\eta\left(\xi^{2}-\sigma^{2}\right) \hat{\psi}_{,}^{2}-\eta\left(1-\eta^{2}\right) \hat{\psi}_{,_{\eta}}^{2}+2 \xi\left(1-\eta^{2}\right) \hat{\psi},_{\xi} \hat{\psi}_{,_{\eta}}\right] .
\end{gathered}
$$

When $\sigma=1$ we have the prolate coordinates, when $\sigma=i$ we have the oblate coordinates, and the case $\sigma=0$ with $\eta=\cos \theta$ and $k=1$ corresponds to the spherical coordinates. As $\hat{\psi}$ satisfies the Laplace's equation, the integrability of the systems (17a) - (18b) is guaranteed. So we can choose any of these equations for find $A$ or $\Lambda$.

\section{A. Solutions in spherical coordinates}

In terms of spherical coordinates $(r, \theta)$ which are related to Weyl coordinates $(\rho, z)$ by

$$
\rho=r \sin \theta, \quad z=r \cos \theta,
$$

with $0 \leq r \leq \infty$ and $-\pi \leq \theta \leq \pi$, the asymptotically flat general solution of Laplace's equation (14) can be written as

$$
\hat{\psi}=-\sum_{n=0}^{\infty} c_{n} \frac{P_{n}(\cos \theta)}{r^{n+1}}
$$

where $c_{n}$ are constants and $P_{n}(\cos \theta)$ are the Legendre polynomials. The magnetic potential obtains from of any the equations (17a) - (17b) in spherical coordinates

$$
A=\int_{\pi}^{\theta} A_{, \theta} d \theta=-b \int_{\pi}^{\theta} r^{2} \sin \theta \hat{\psi},{ }_{r} d \theta
$$

where the integral limits are chosen by requiring that the function $A$ to be regular on the axis of symmetry. So

$$
\begin{aligned}
A & =-b \sum_{n=0}^{\infty} c_{n}(n+1) \frac{1}{r^{n}} \int_{\pi}^{\theta} \sin \theta P_{n}(\cos \theta) d \theta \\
& =b \sum_{n=0}^{\infty} c_{n}(n+1) \frac{1}{r^{n}} \int_{\pi}^{\theta} P_{n}(\cos \theta) d(\cos \theta) .
\end{aligned}
$$

With the change of variable $y=\cos \theta$ the above expression takes the form

$$
A=b \sum_{n=0}^{\infty} c_{n}(n+1) \frac{1}{r^{n}} \int_{-1}^{y} P_{n}(y) d y
$$

and using the identity [75]

$$
\int_{-1}^{y} P_{n}(y) d y=\frac{1}{2 n+1}\left(P_{n+1}(y)-P_{n-1}(y)\right), \quad n \geq 0, \quad P_{-1}=-1,
$$


we obtain

$$
A=b \sum_{n=0}^{\infty} \frac{c_{n}(n+1)}{(2 n+1) r^{n}}\left[P_{n+1}(\cos \theta)-P_{n-1}(\cos \theta)\right] .
$$

Finally, using the identity [6]

$$
P_{n+1}(\cos \theta)-P_{n-1}(\cos \theta)=\frac{2 n+1}{n+1}\left(\cos \theta P_{n}(\cos \theta)-P_{n-1}(\cos \theta)\right),
$$

one finds that the magnetic potential can be written as

$$
A=b \sum_{n=0}^{\infty} \frac{c_{n}}{r^{n}}\left[\cos \theta P_{n}(\cos \theta)-P_{n-1}(\cos \theta)\right]
$$

The same above procedure is carried out to find $\Lambda[73]$ and we obtain

$$
\begin{aligned}
\Lambda & =\int_{\pi}^{\theta} \hat{\Lambda}_{\theta} d \theta \\
& =-\sum_{l, m=0}^{\infty} \frac{c_{l} c_{m}(l+1)(m+1)}{(l+m+2) r^{l+m+2}}\left[P_{l} P_{m}-P_{l+1} P_{m+1}\right] .
\end{aligned}
$$

Since $r=\sqrt{\rho^{2}+z^{2}}$ the solutions have only a true singularity at the point $\rho=0, z=0$. Hence the solutions are regular on the axis of symmetry, except at the origin. Such as singularity could be interpreted as the inside some kind of black hole and the solutions as the gravitational field produced by such source. The Curzon-Scott-Szekeres black holes [77, 78] provides an example of this type of spacetimes. However, the problems associated with the complicated structure of the singularity in these space-times can be avoided by considering the solution to represent the exterior field of some finite source, e.g, representing the field of a disk (section III). In addition, since $|\cos (\theta)| \leq 1$ and $\left|P_{n}(\cos \theta)\right| \leq 1$ [76] the magnetic potential A (25) tends to zero at infinity only for $n \geq 1$. Hence, the solutions are asymptotically flat only for $n \geq 1$, i.e., if the coefficient $c_{0}$ vanishes. On the other hand, for the line element (11), in terms of the spherical coordinates (19),

$$
d s^{2}=-e^{2 \psi} d t^{2}+e^{-2 \psi}\left[r^{2} \sin ^{2} \theta d \varphi^{2}+e^{2 \Lambda}\left(d r^{2}+r^{2} d \theta^{2}\right)\right]
$$

the total mass 79

$$
m=-\frac{1}{4 \pi} \lim _{r \rightarrow \infty} \int_{S_{r}} K^{a ; b} d S_{a b}
$$

(where $S_{r}$ is a coordinate sphere with radius $r$ and $K=\partial t$ is the static Killing vector) for an asymptotically flat solution is given by

$$
m=-\lim _{r \rightarrow \infty}\left(r^{2} \psi_{, r}\right)
$$

For a seed solution of the form (20) we have

$$
\hat{m}=-\lim _{r \rightarrow \infty}\left(r^{2} \hat{\psi}_{, r}\right)=-c_{0}
$$

From (15a)

$$
\psi_{, r}=\hat{\psi}_{, r}\left[\frac{(1+a) \mathrm{e}^{-\hat{\psi}}-(1-a) \mathrm{e}^{\hat{\psi}}}{(1+a) \mathrm{e}^{-\hat{\psi}}+(1-a) \mathrm{e}^{\hat{\psi}}}\right],
$$

so that using (30) and the fact that $\psi \rightarrow 0$ for $r \rightarrow \infty$ we obtain

$$
m=-\lim _{r \rightarrow \infty}\left(r^{2} \hat{\psi}_{, r}\right) \lim _{r \rightarrow \infty}\left[\frac{(1+a) \mathrm{e}^{-\hat{\psi}}-(1-a) \mathrm{e}^{\hat{\psi}}}{(1+a) \mathrm{e}^{-\hat{\psi}}+(1-a) \mathrm{e}^{\hat{\psi}}}\right]=\hat{m} a=-a c_{0} .
$$


Now if one chooses $c_{0}=0$ in order to guarantee asymptotical flatness this leads to $m=0$ for arbitrary coefficients $c_{1}, c_{2}$, etc. These parameters contribute to higher multipole moments [80] but not to the mass. According to the positive mass theorem this imply that the spacetime must be singular or Minkowski space. So when the magnetic field is present, i.e., if $b \neq 0$, these solutions are massless in the asymptotically flat case. Moreover, since the expression for the mass (28) is invariant, this result is true for any coordinate system. Therefore, in presence of magnetic field these solutions are either singular, not asymptotically flat or massless.

For $n=0$ we have that

$$
\hat{\psi}=-\frac{m}{r}
$$

where we have chosen $c_{0}=m$. Therefore, the exact solution of Einstein-Maxwell field equations for $n=0$ is

$$
\begin{aligned}
e^{\psi} & =\frac{2}{(1+a) e^{m / r}+(1-a) e^{-m / r}}, \\
\Lambda & =-\frac{m^{2}}{2 r^{2}} \sin ^{2} \theta \\
A & =b m(\cos \theta+1) .
\end{aligned}
$$

This solution is the magnetized version of the Chazy-Curson vacuum solution [42, 81, 82]. The solution is regular on the axis of symmetry but not is asymptotically flat. Therefore, this solution represents the exterior gravitational field of a source of finite mass in the presence of a magnetic field spread throughout the space (infinite).

We now consider the sum of the first and second terms $n=0$ and $n=2$ of series (20). In this case we have

$$
\hat{\psi}=-\frac{c_{0}}{r}-\frac{c_{2}}{2 r^{3}}\left(3 \cos ^{2} \theta-1\right)
$$

and the general solutions in spherical coordinates in this case is

$$
\begin{aligned}
A= & b c_{0}(\cos \theta+1)-\frac{3}{2} b c_{2} \frac{1}{r^{2}} \cos \theta \sin ^{2} \theta \\
\Lambda= & -\frac{1}{8 r^{6}} \sin ^{2} \theta\left[3 c_{2} \cos ^{2} \theta\left(25 c_{2} \cos ^{2} \theta+10 c_{0} r^{2}-14 c_{2}\right)\right. \\
& \left.+4 c_{0}^{2} r^{4}-6 c_{0} c_{2} r^{2}+3 c_{2}^{2}\right]
\end{aligned}
$$

and $\psi$ is given by (1).

\section{B. Solutions in prolate spheroidal coordinates}

In terms of the prolate coordinates $(x, y)$ which are related to Weyl coordinates $(\rho, z)$ by

$$
\begin{aligned}
\rho^{2} & =k^{2}\left(x^{2}-1\right)\left(1-y^{2}\right), \\
z & =k x y
\end{aligned}
$$

with $x \geq 1,-1<y<1$, and $k$ a constant, the asymptotically flat general solution of Laplace's equation (14) can be written as

$$
\hat{\psi}=-\sum_{n=0}^{\infty} c_{n} Q_{n}(x) P_{n}(y)
$$

where $c_{n}$ are constants and the $Q_{n}(x)$ are the Legendre functions of the second kind

$$
Q_{n}(x)=\sum_{s=0}^{\infty} \frac{2^{n}(n+s) !(n+2 s) !}{s !(2 n+2 s+1) !} x^{-2 s-n-1} .
$$

From (17a) - (17b) in prolate coordinates, we obtain

$$
A=-k b\left(x^{2}-1\right) \sum_{n=0}^{\infty} c_{n} Q_{n}^{\prime}(x) \int_{-1}^{y} P_{n}(y) d y,
$$


where the integral limits are chosen by requiring that the function $A$ to be regular on the axis of symmetry. Using the identities (22) and (24), we have that

$$
A=-k b\left(x^{2}-1\right) \sum_{n=0}^{\infty} \frac{c_{n}}{n+1} Q_{n}^{\prime}(x)\left[y P_{n}(y)-P_{n-1}(y)\right] .
$$

Since $Q_{n}(x)$ diverges for $x \rightarrow 1$, the resulting solutions are singular at $x=1$, corresponding to $\rho=0,-1<y<1$, i.e., on a part of the symmetric. In the vacuum these solutions have been interpreted by several authors as distorted static black holes [83]. Using the definition for $Q_{n}(x)$ (39), the recurrence relation

$$
\left(x^{2}-1\right) Q_{n}^{\prime}(x)=(n+1)\left[Q_{n+1}(x)-x Q_{n}(x)\right]
$$

and the fact that $Q_{n}(\infty)=0$ we find that in the infinity $(x \rightarrow \infty)$

$$
\lim _{x \rightarrow \infty}\left(x^{2}-1\right) Q_{n}^{\prime}(x)=\left\{\begin{array}{ll}
-1, & n=0 \\
0, & n \geq 1
\end{array} .\right.
$$

Therefore, the magnetic potential A (41) tends to zero at infinity,

$$
\lim _{x \rightarrow \infty} A=0
$$

only for $n \geq 1$. Hence, the asymptotically flat solutions are those for which $n \geq 1$, i.e., if the coefficient $c_{0}$ vanishes. Therefore, as was discussed above, in presence of magnetic field these solutions are either singular, not asymptotically flat or massless.

For $n=0$ we have

$$
\hat{\psi}=\frac{c_{0}}{2} \ln \left(\frac{x-1}{x+1}\right) .
$$

and the magnetic potential is

$$
\begin{aligned}
A & =-k b c_{0}\left(x^{2}-1\right) Q_{0}^{\prime}(x)\left[y P_{0}(y)-P_{-1}(y)\right] \\
& =-k b c_{0}\left(x^{2}-1\right)(y+1) Q_{0}^{\prime}(x)
\end{aligned}
$$

Using

$$
\begin{aligned}
& Q_{0}(x)=\frac{1}{2} \ln \left(\frac{x+1}{x-1}\right), \\
& Q_{0}^{\prime}(x)=-\frac{1}{\left(x^{2}-1\right)},
\end{aligned}
$$

we obtain

$$
A=k b c_{0}(y+1)
$$

So the solution in prolate spheroidal coordinates for $n=0$ is

$$
\begin{aligned}
e^{\psi} & =\frac{2\left(x^{2}-1\right)^{c_{0} / 2}}{(1+a)(x+1)^{c_{0}}+(1-a)(x-1)^{c_{0}}}, \\
\Lambda & =\frac{c_{0}^{2}}{2} \ln \left[\frac{x^{2}-1}{x^{2}-y^{2}}\right] \\
A & =k b c_{0}(y+1) .
\end{aligned}
$$

This solution is the magnetized version of the Zipoy-Voorhees vacuum solution [42, 84, 85]. For $b=0$ and $c_{0}=1$ we have the Schwarzschild solution [86] and the case $b=0$ and $c_{0}=2$ corresponds to the metric of Darmois [73]. The solution is regular on the axis of symmetry but is not asymptotically flat. Therefore, this solution also represents the gravitational field outside a source of finite mass in the presence of a magnetic field spread throughout the space (infinite).

For the terms $n=0$ and $n=2$ we have that

$$
\hat{\psi}=c_{0} \frac{1}{2} \ln \left(\frac{x-1}{x+1}\right)+\frac{1}{2} c_{2}\left(3 y^{2}-1\right)\left[\frac{1}{4}\left(3 x^{2}-1\right) \ln \left(\frac{x-1}{x+1}\right)+\frac{3}{2} x\right],
$$


and for the second term of the summation $n=2$ the magnetic potential is

$$
\begin{aligned}
A(n=2) & =-k b\left(x^{2}-1\right) c_{2} \frac{1}{2} y\left(1-y^{2}\right) Q_{2}^{\prime}(x), \\
& =-\frac{1}{4} k b c_{2} y\left(1-y^{2}\right)\left[3 x\left(x^{2}-1\right) \ln \left(\frac{x-1}{x+1}\right)+6 x^{2}-4\right] .
\end{aligned}
$$

Therefore the solution in prolate spheroidal coordinate in this case is

$$
\begin{aligned}
A= & k b c_{0}(y+1)-\frac{1}{4} k b c_{2} y\left(1-y^{2}\right)\left[3 x\left(x^{2}-1\right) \ln \left(\frac{x-1}{x+1}\right)+6 x^{2}-4\right] \\
\Lambda= & \frac{9}{64} c_{2}^{2}\left(x^{2}-1\right)\left(y^{2}-1\right)\left(9 x^{2} y^{2}-x^{2}+1-y^{2}\right)\left[\ln \left(\frac{x-1}{x+1}\right)\right]^{2} \\
& +\frac{3}{16} c_{2} x\left(y^{2}-1\right)\left(27 c_{2} x^{2} y^{2}-3 c_{2} x^{2}-21 c_{2} y^{2}+5 c_{2}+8 c_{0}\right) \ln \left(\frac{x-1}{x+1}\right) \\
& +\frac{1}{2}\left(c_{0}+c_{2}\right)^{2} \ln \left(\frac{x^{2}-1}{x^{2}-y^{2}}\right) \\
& +\frac{3}{16} c_{2}\left(y^{2}-1\right)\left(-12 c_{2} y^{2}+27 c_{2} x^{2} y^{2}-3 c_{2} x^{2}+4 c_{2}+16 c_{0}\right),
\end{aligned}
$$

and $\psi$ is given by (15a). In the absence of magnetic field $b=0$ and $c_{0}=1$, we have the Erez-rosen vacuum metric [87] so that the case $b \neq 0$ corresponds to a magnetized Erez-rosen metric.

\section{Solutions in oblate spheroidal coordinates}

In terms of the oblate coordinates $(u, v)$ which are related to Weyl coordinates $(\rho, z)$ by

$$
\begin{aligned}
\rho^{2} & =k^{2}\left(u^{2}+1\right)\left(1-v^{2}\right), \\
z & =k u v,
\end{aligned}
$$

with $u \geq 0,-1<v<1$, and $k$ a constant, the asymptotically flat general solution of Laplace's equation (14) can be written as

$$
\hat{\psi}=-\sum_{n=0}^{\infty} c_{n} q_{n}(u) P_{n}(v)
$$

where $c_{n}$ are constants and

$$
q_{n}(u)=i^{n+1} Q_{n}(i u)
$$

Again requiring that the solution to be regular on the axis of symmetry, it follows that

$$
\begin{aligned}
A & =-k b\left(u^{2}+1\right) \sum_{n=0}^{\infty} c_{n} q_{n}^{\prime}(u) \int_{-1}^{v} P_{n}(v) d v \\
& =-k b\left(u^{2}+1\right) \sum_{n=0}^{\infty} \frac{c_{n}}{2 n+1} q_{n}^{\prime}(u)\left[P_{n+1}(v)-P_{n-1}(v)\right],
\end{aligned}
$$

and using the identities (22) y (24) we obtain the following expression for the magnetic potential

$$
A=-k b\left(u^{2}+1\right) \sum_{n=0}^{\infty} \frac{c_{n}}{n+1} q_{n}^{\prime}(u)\left[v P_{n}(v)-P_{n-1}(v)\right],
$$

and again $\psi$ is given by (15a). In the absence of magnetic field $b=0$ and for $n$ even this solutions correspond to the Morgan-Morgan vacuum solutions [25] which represent the exterior gravitational field produced by a finite 
disklike source. So we can call above solutions magnetized Morgan-Morgan solutions. Similarly to the prolate case, the asymptotically flat solutions are those for which $n \geq 1$, i.e., if the coefficient $c_{0}$ vanishes. Hence, as was discussed above, in presence of magnetic field these solutions are either singular, not asymptotically flat or massless.

For $n=0$ we have

$$
\hat{\psi}=-c_{0} \cot ^{-1}(u)
$$

and the potential magnetic is given by

$$
A=-k b c_{0}\left(u^{2}+1\right) q_{0}^{\prime}(u)(v+1),
$$

with

$$
\begin{aligned}
& q_{n}^{\prime}(u)=i^{n+1} Q_{n}^{\prime}(i u), \\
& q_{0}^{\prime}(u)=-\frac{1}{u^{2}+1} .
\end{aligned}
$$

Hence that for $n=0$ the solutions is

$$
\begin{aligned}
e^{\psi} & =\frac{2}{(1+a) e^{c_{0} \cot ^{-1} u}+(1-a) e^{-c_{0} \cot ^{-1} u},} \\
\Lambda & =-\frac{c_{0}^{2}}{2} \ln \left[\frac{u^{2}+1}{u^{2}+v^{2}}\right], \\
A & =k b c_{0}(v+1) .
\end{aligned}
$$

This solution is the magnetized version of the Bonnor-Sackfield vacuum solution or zero order Morgan-Morgan solution [42, 88]. The solution is regular on the axis of symmetry but is not asymptotically flat and represents the exterior gravitational field of finite disklike source in the presence of a magnetic field spread throughout the space (infinite).

For the terms $n=0$ and $n=2$ of series (55) we have

$$
\hat{\psi}=-c_{0} \cot ^{-1}(u)-\frac{1}{4} c_{2}\left(3 v^{2}-1\right)\left[\left(3 u^{2}+1\right) \cot ^{-1}(u)-3 u\right] .
$$

For the second term of the summation we have

$$
\begin{aligned}
A & =-k b \frac{c_{2}}{5}\left(u^{2}+1\right) q_{2}^{\prime}(u)\left[v P_{2}(v)-P_{1}(v)\right] \\
& =\frac{1}{2} k b c_{2}\left(u^{2}+1\right) v\left(1-v^{2}\right) q_{2}^{\prime}(u) \\
& =\frac{1}{2} k b c_{2} v\left(1-v^{2}\right)\left[3 u\left(u^{2}+1\right) \cot ^{-1}(u)-3 u^{2}-2\right] .
\end{aligned}
$$

Finally, the solutions is

$$
\begin{aligned}
A= & k b c_{0}(v+1)+\frac{1}{2} k b c_{2} v\left(1-v^{2}\right)\left[3 u\left(u^{2}+1\right) \cot ^{-1}(u)-3 u^{2}-2\right], \\
\Lambda= & -\frac{9}{16 k^{2}} c_{2}^{2} \rho^{2}\left(9 u^{2} v^{2}-u^{2}+v^{2}-1\right) \cot ^{-1}(u)^{2} \\
& +\frac{3}{8} c_{2} u\left(1-v^{2}\right)\left(27 c_{2} u^{2} v^{2}-3 c_{2} u^{2}+21 c_{2} v^{2}-5 c_{2}+8 c_{0}\right) \cot ^{-1}(u) \\
& +\frac{1}{2}\left(c_{2}-c_{0}\right)^{2} \ln \left(\frac{u^{2}+v^{2}}{1+u^{2}}\right) \\
& -\frac{3}{16} c_{2}\left(1-v^{2}\right)\left(12 c_{2} v^{2}+27 c_{2} u^{2} v^{2}-3 c_{2} u^{2}-4 c_{2}+16 c_{0}\right),
\end{aligned}
$$

and again $\psi$ is given by (15a). In the absence of magnetic field $b=0$ and $c_{0}=c_{2}$, this solution corresponds the first order Morgan-Morgan vacuum solution. Therefore the case $b \neq 0$ corresponds to a magnetized first order Morgan-Morgan solution. 


\section{Solutions in generalized spheroidal coordinates}

We can reunite the three general solutions presented above in a single family by considering generalized spheroidal coordinates $(\xi, \eta)$ which are a generalization of the spherical coordinates, prolate and oblate. The asymptotically flat general solution of Laplace's equation (14) can be written in this coordinates

$$
\hat{\psi}(\varepsilon, \eta)=-\sum_{n=0}^{\infty} \frac{c_{n}}{\sigma^{n+1}} Q_{n}(\varepsilon) P_{n}(\eta)
$$

where $c_{n}$ are constants and $\varepsilon=\xi / \sigma$. The expressions corresponding to the metric functions and the magnetic potential are obtained in the same way as the previous cases, that is

$$
\begin{aligned}
& A(\varepsilon, \eta)=\int_{-1}^{\eta} A_{, \eta} d \eta \\
& \Lambda(\varepsilon, \eta)=\int_{-1}^{\eta} \hat{\Lambda}_{, \eta} d \eta .
\end{aligned}
$$

So the magnetic potential $A$ is

$$
A=-k b\left(\xi^{2}-\sigma^{2}\right) \sum_{n=0}^{\infty} \frac{c_{n}}{(n+1) \sigma^{n+1}} Q_{n}^{\prime}(\varepsilon)\left[\eta P_{n}(\eta)-P_{n-1}(\eta)\right]
$$

and the metric function $\Lambda$ is given by $[80,89]$

$$
\Lambda=\sum_{n, m=0}^{\infty} \frac{c_{n} c_{m}}{\sigma^{n+m+1}} \Gamma^{m n}
$$

where $Q_{n}^{\prime}(\xi)$ is the total derivate of $Q_{n}(\xi)$ with respect to $\xi$ and

$$
\begin{aligned}
\Gamma^{m n}= & \frac{1}{2} \ln \left[\frac{\varepsilon^{2}-1}{\varepsilon^{2}-\eta^{2}}\right]+\left(k_{n}+k_{m}-2 k_{n} k_{m}\right) \ln \left[\frac{\varepsilon+\eta}{\varepsilon-1}\right] \\
& +\left(\varepsilon^{2}-1\right)\left[\varepsilon\left(A_{n, m} Q_{n}^{\prime} Q_{m}+A_{m, n} Q_{m}^{\prime} Q_{n}\right)-C_{n, m} Q_{n} Q_{m}\right] \\
& +\left(\varepsilon^{2}-1\right)\left[\left(1-k_{n}\right) S_{m}+k_{n} S_{m+1}-\frac{k_{n}}{m+1}\left(P_{m}-(-1)^{m} Q_{m}^{\prime}\right)\right] \\
& +\left(\varepsilon^{2}-1\right)^{2}\left[Q_{m} B_{m, n}-Q_{m}^{\prime} A_{m, n}+\frac{1}{n+1} A_{m, n} Q_{m}^{\prime} Q_{n}^{\prime}\right]
\end{aligned}
$$

with

$$
k_{l}=\left\{\begin{array}{l}
1 \text { for } l \text { even } \\
0 \text { for } l \text { odd }
\end{array}\right.
$$

and where $A_{n, m}, B_{n, m}, C_{n, m}$ and $S_{n}$ are

$$
\begin{aligned}
A_{n, m}+A_{m, n} & =P_{n} P_{m}-(-1)^{n+m} \int_{-1}^{\eta} P_{m}^{\prime} P_{n} d \eta \\
B_{n, m}=B_{n, m} & =\int_{-1}^{\eta} P_{m}^{\prime} P_{n} d \eta \\
C_{n, m} & =C_{n, m}=\int_{-1}^{\eta} \eta P_{m}^{\prime} P_{n} d \eta \\
S_{n} & =\sum_{k=0}^{(n-2) / 2}\left[\frac{1}{n-2 k}+\frac{1}{n-2 k-1}\right]\left(P_{n-2 k-1}+(-1)^{n+1}\right) Q_{n-2 k-1}^{\prime} .
\end{aligned}
$$

Finally, the function $\psi$ is given by equation (15a), and with $b \neq 0$ the solutions are asymptotically flat for $n \geq 1$, i.e., if the coefficient $c_{0}$ is chosen equal to zero. So in presence of magnetic field all discussed solutions are either singular, not asymptotically flat or massless. 


\section{RELATIVISTIC THIN DISKS}

In order to obtain a solution of the Einstein-Maxwell equations (2a) - 2c) representing a thin disk at $z=0$ with current, we assume that the components of the metric tensor and of the electromagnetic potential are continuous across the disk, but with first derivatives discontinuous on the plane $z=0$, with discontinuity functions

$$
\begin{aligned}
b_{a b} & =\left.g_{a b, z}\right|_{z=0^{+}}-\left.g_{a b, z}\right|_{z=0^{-}}=\left.2 g_{a b, z}\right|_{z=0^{+}}, \\
a_{a} & =\left.A_{a, z}\right|_{z=0^{+}}-\left.A_{a, z}\right|_{z=0^{-}}=\left.2 A_{a, z}\right|_{z=0^{+}} .
\end{aligned}
$$

By using the distributional approach 90 92] or the junction conditions on the extrinsic curvature of thin shells 93, 94], the Einstein-Maxwell equations yield an energy-momentum tensor $T_{a b}=T_{a b}^{\mathrm{elm}}+T_{a b}^{\mathrm{mat}}=T_{a b}^{\mathrm{elm}}+Q_{a b} \delta(z)$, and a current density $J_{a}=j_{a} \delta(z)=-e^{2(\psi-\Lambda)} a_{a} \delta(z)$, where $\delta(z)$ is the usual Dirac function with support on the disk, $T_{a b}^{\mathrm{elm}}$ is the electromagnetic tensor defined in Eq. (2b), $j_{a}$ is the current density on the plane $z=0$, and

$$
Q_{b}^{a}=\frac{1}{2}\left\{b^{a z} \delta_{b}^{z}-b^{z z} \delta_{b}^{a}+g^{a z} b_{b}^{z}-g^{z z} b_{b}^{a}+b_{c}^{c}\left(g^{z z} \delta_{b}^{a}-g^{a z} \delta_{b}^{z}\right)\right\}
$$

is the distributional energy-momentum tensor. The "true" surface energy-momentum tensor (SEMT) of the disk, $S_{a b}$, and the "true" surface current density, $j_{a}$, can be obtained through the relations

$$
\begin{aligned}
S_{a b} & =\int T_{a b}^{\mathrm{mat}} d s_{n}=e^{\Lambda-\psi} Q_{a b}, \\
j_{a} & =\int J_{a} d s_{n}=e^{\Lambda-\psi} j_{a},
\end{aligned}
$$

where $d s_{n}=\sqrt{g_{z z}} d z$ is the "physical measure" of length in the direction normal to the disk. For the metric (11), the nonzero components of $S_{a}^{b}$ are

$$
\begin{aligned}
& S_{0}^{0}=2 e^{\psi-\Lambda}\left\{\Lambda,{ }_{z}-2 \psi,{ }_{z}\right\}, \\
& S_{1}^{1}=2 e^{\psi-\Lambda} \Lambda, z,
\end{aligned}
$$

and the only nonzero component of the current density $j_{a}$ in the magnetostatic case is

$$
j_{\varphi}=-\frac{1}{2 \pi} e^{\psi-\Lambda} A_{, z}
$$

where all the quantities are evaluated at $z=0^{+}$.

In order to give physical significance to the components of the energy-momentum tensor $S_{a}^{b}$ and the electric current density $j_{a}$ we project them onto the orthonormal tetrad $\mathrm{e}_{(a)}{ }^{b}=\left\{V^{b}, W^{b}, X^{b}, Y^{b}\right\}$, where

$$
\begin{aligned}
V^{a} & =e^{-\psi}(1,0,0,0), \\
W^{a} & =\frac{e^{\psi}}{\rho}(0,1,0,0), \\
X^{a} & =e^{\psi-\Lambda}(0,0,1,0), \\
Y^{a} & =e^{\psi-\Lambda}(0,0,0,1) .
\end{aligned}
$$

In terms of this tetrad (or observer with four-velocity $V^{a}$ ) the surface energy density $\epsilon$, the azimuthal pressure $p_{\varphi}$, and the azimuthal current density $j$ of the disk are given by

$$
\epsilon=-S_{0}^{0}, \quad p_{\varphi}=S_{1}^{1}, \quad j=W^{1} j_{1} .
$$

Finite thin disks can be obtained introducing oblate spheroidal coordinates, which are naturally adapted to a disk source. These solutions, in the vacuum and static case, correspond to the Morgan and Morgan solutions [25]. In the case of the magnetized Morgan-Morgan solutions, following the reference [25], for $n$ even the metric functions are continuous across the disk but its first derivatives are discontinuous in the direction normal to the disk, which can be interpreted as a finite thin disk located at $z=0$ and $0 \leq \rho \leq 1$. However, since for $n$ even the function $A$ is a odd 
polynomial of $v$, the opposite occurs with the magnetic potential, i.e, it is discontinuous across the disk but its first derivatives are continuous in the direction normal to the disk, so that electric current density also is zero on the disk and in consequence the source of the magnetic field is non planar but of a different origin such as a remnants or fossil magnetic field [62], or can come from external sources, such as the presence of a nearby magnetars or neutron stars. Indeed on the disk the Maxwell equations $\partial_{b} \bar{F}^{a b}=4 \pi \bar{J}^{a}$, where 'bar' denotes multiplication by $\sqrt{-g}$, are given by

$$
-4 \pi \bar{j}_{\varphi} \delta(z)=\partial_{z} g^{z z} \bar{A}_{, z}+\partial_{\rho} g^{\rho \rho} \bar{A}_{, \rho}
$$

where $\delta(z)$ is the usual Dirac Function with support on the disk. Integrating through the disk we obtain

$$
\begin{aligned}
-4 \pi \bar{j}_{\varphi} & =\int_{z=0_{-}}^{z=0_{+}} \partial_{z} g^{z z} \bar{A}_{, z} d z+\int_{z=0_{-}}^{z=0_{+}} \partial_{\rho} g^{\rho \rho} \bar{A}_{, \rho} d z \\
& =\left.g^{z z} \bar{A}_{, z}\right|_{z=0_{-}} ^{z=0_{+}}+\partial_{\rho} g^{\rho \rho} \partial_{\rho} \int_{z=0_{-}}^{z=0_{+}} \bar{A} d z \\
& =0
\end{aligned}
$$

where the first term on the right-hand side vanishes due to the continuity of the metric and $A_{, z}$ and the second term from discontinuity of $A$, or in other words as $A$ is an odd polynomial function of $v$ its integral through the disk is even and hence continuous, so that to the evaluate at the limits of integration expression vanishes. Thus we can interpret this solutions as the exterior gravitational field of a finite dislike source immersed in a magnetic field. For completeness, we analysis the electrostatic case, i.e., for $\Phi$ real. The electric potential $\phi$ is given by

$$
\phi=\frac{p\left(e^{-\hat{\psi}}-e^{\hat{\psi}}\right)}{(1+a) e^{-\hat{\psi}}+(1-a) e^{\hat{\psi}}},
$$

where $a=\sqrt{1+p^{2}}$, being $p$ the parameter that controls the electric field, and the only non-zero component of the current density $j_{a}$ on the plane $z=0$ is

$$
j_{t}=-\frac{1}{2 \pi} e^{\psi-\Lambda} \phi_{, z}
$$

whereas in terms of the tetrad (179) the electric charge density $\sigma$ is given by

$$
\sigma=-V^{0} j_{0}
$$

We see that just as metric the electric potential $\phi$ is a even function of $v$ and in consequence the current density in the disk is nonzero. Therefore, this solutions can be interpreted as the gravitational field of a finite charged disk.

In order to study the behavior of the main physical quantities associated with these disks we perform a graphical analysis of them for magnetized first order Morgan-Morgan finite disks. In Fig. 1 we show the energy density $\epsilon$ and the azimuthal pressure $p_{\varphi}$ with $c_{0}=c_{2}=0.4$ and for values of magnetic field parameter $b=0$ (dashed curves), 0.5 , 1 , and 2 (bottom curves), as functions of $\rho$. We see that the energy density presents a maximum at $\rho=0$ and then decreases with $\rho$. We also see that the presence of magnetic field decreases the energy density at the central region of the disk and later increases it. We can observe that the pressure increases rapidly as one moves away from the disk center, reaches a maximum and later decreases. We also observe that the magnetic field decreases the pressure everywhere on the disk. The graph also show that the disk's surface energy density is always positive in concordance with the weak energy condition, as well as the stress in azimuthal direction (pressure). The strong energy condition, $\epsilon+p_{\varphi}>0$, is also satisfied. These properties characterize a fluid made of matter with the usual gravitational attractive property. In addition, in Fig. 1(c) we show, in the electrostatic case, the surface electric charge density $\sigma$ for values of electric field parameter $p=0.2$ (dashed curve), 0.5, and 1 (top curve) and the same values of $c_{0}$ and $c_{2}$. We observe a greater concentration of electric charge near the rim of the disk and that this increases as we increase the electric field.

Exact solutions which represent the field of a disk also can be obtained using the well known "displace, cut and reflect" method that was first used by Kuzmin 95] and Toomre 96 to constructed Newtonian models of disks, and later extended to general relativity [33, 34, 36, 37]. Given a solution of the Einstein-Maxwell equation, this procedure is mathematically equivalent to apply the transformation $z \rightarrow|z|+z_{0}$, with $z_{0}$ constant, on that solution. However, this disks are essentially of infinite extension and the field not correspond exactly to the metric with which we started. These solutions can be called in our case magnetized Weyl type solutions. The reflection symmetry with respect to the plane of disk implies that in this case $A$ is continuous across the disk and its first derivative discontinuous in the 
direction normal to the disk, so that electric current density is nonzero on the disk and in consequence the source of the magnetic field is planar.

In Figs. 2 - 4 we show, as functions of $\rho$, the energy density $\epsilon$, the azimuthal pressure $p_{\varphi}$ and surface azimuthal electric current density $j$ for magnetized Weyl type disks of infinite extension corresponding to the lineal combination of the first and second terms of the series in the three families of solutions considered. Since the surface energy density decreases rapidly one can to define a cut off radius and, in principle, to consider these disks as finite. Anyway all the physical quantities present a similar behavior to the previous case.

\section{MOTION OF CHARGED PARTICLES AROUND MAGNETIZED WEYL FIELDS}

The relativistic Lagrangian for a test particle in presence of a gravitational and magnetic field is given by

$$
\mathcal{L}=\frac{1}{2} g_{a b} \dot{x}^{a} \dot{x}^{b}+\tilde{e} A \dot{\varphi}
$$

where $\tilde{e}=e / m$ is the specific charge of the particle and the overdot denotes derivate with respect to the proper time $s$. For magnetized Weyl fields we have two constants of motion

$$
\begin{aligned}
& E=-p_{t} / m=-g_{t t} \dot{t}, \\
& L=p_{\varphi} / m=g_{\varphi \varphi} \dot{\varphi}+\tilde{e} A,
\end{aligned}
$$

where $E$ represents the relativistic specific energy and $L$ the specific angular momentum.

For circular orbits and from symmetry of the field the equation for the electrogeodesic motion of the particle is given by

$$
\frac{1}{2} g_{a b, \rho} u^{a} u^{b}=-\tilde{e} F_{\rho a} u^{a}
$$

where $u^{a}$ is the 4 -velocity of particles with respect a the coordinates frame. For equatorial circular orbits $u^{a}$ has components $u^{a}=u^{0}(1, \omega, 0,0)$ where $\omega=u^{1} / u^{0}=\frac{d \varphi}{d t}$ is the angular velocity of the test particles, and in the case magnetostatic the equation of motion reads

$$
\frac{1}{2} u^{0}\left(g_{\varphi \varphi, \rho} \omega^{2}+g_{t t, \rho}\right)=-\tilde{e} A_{, \rho} \omega,
$$

where $u^{0}$ obtains normalizing $u^{a}$, that is requiring $g_{a b} u^{a} u^{b}=-1$. Thus with

$$
\left(u^{0}\right)^{2}=-\frac{1}{g_{\varphi \varphi} \omega^{2}+g_{t t}}
$$

the equation of the electrogeodesic takes the form

$$
\bar{A}\left(\omega^{2}\right)^{2}+\bar{B} \omega^{2}+\bar{C}=0
$$

where

$$
\begin{aligned}
\bar{A} & =g_{\varphi \varphi, \rho}^{2}+4 \tilde{e}^{2} g_{\varphi \varphi} A_{, \rho}^{2} \\
& =4 \rho^{2} e^{-2 \psi}\left[\left(1-\rho \psi_{, \rho}\right)^{2} e^{-2 \psi}+\tilde{e}^{2} A_{, \rho}^{2}\right], \\
\bar{B} & =2 g_{t t, \rho} g_{\varphi \varphi, \rho}+4 \tilde{e}^{2} g_{t t} A_{, \rho}^{2} \\
& =-4\left[2 \rho \psi_{, \rho}\left(1-\rho \psi_{, \rho}\right)+\tilde{e}^{2} A_{, \rho}^{2} e^{2 \psi}\right], \\
\bar{C} & =g_{t t, \rho}^{2}=4 \psi_{, \rho}^{2} e^{4 \psi} .
\end{aligned}
$$

Therefore, the angular velocity $\omega$ is given by

$$
\omega^{2}=\frac{-\bar{B} \pm \sqrt{D}}{2 \bar{A}}
$$

where

$$
D=\bar{B}^{2}-4 \bar{A} \bar{C}=16 \tilde{e}^{2} A_{, \rho}^{2} e^{2 \psi}\left[4 \rho \psi_{, \rho}\left(1-2 \rho \psi_{, \rho}\right)+\tilde{e}^{2} A_{, \rho}^{2} e^{2 \psi}\right] \geq 0
$$


and positive sign corresponds to the direct orbits (or co-rotating with $L>0$ ) and the negative sign to the retrograde orbits (or counter-rotating with $L<0$ ). From (91) and (94) we find

$$
\begin{aligned}
E & =-g_{t t} u^{0}, \\
L & =g_{\varphi \varphi} \omega u^{0}+\tilde{e} A .
\end{aligned}
$$

With respect to the orthonormal tetrad (79) the 3-velocity has components

$$
v^{(i)}=\frac{\mathrm{e}^{(i)}{ }_{a} u^{a}}{\mathrm{e}^{(0)}{ }_{b} u^{b}} .
$$

For equatorial circular orbits the only nonvanishing velocity components is given by

$$
\left(v^{(\varphi)}\right)^{2}=v_{c}^{2}=-\frac{g_{\varphi \varphi}}{g_{t t}} \omega^{2}=\rho^{2} \mathrm{e}^{-4 \psi} \omega^{2}
$$

which is the circular velocity of the particle as seen by an observer at infinity. In fact when $\rho \rightarrow \infty, \psi \rightarrow 0$ and $v_{c}^{2}=\rho^{2} \omega^{2}=\rho \frac{d \phi}{d \rho}$, where $\phi$ is the Newtonian gravitational potential, and $v_{c}$ is the circular velocity, which represents the velocity of a test particle in a circular orbit at radius $\rho$. For neutral particles $\tilde{e}=0$ we have

$$
v_{c}^{2}=\frac{\rho \psi, \rho}{1-\rho \psi, \rho}
$$

with

$$
\psi_{, \rho}=\hat{\psi}_{, \rho}\left[\frac{(1+a) \mathrm{e}^{-\hat{\psi}}-(1-a) \mathrm{e}^{\hat{\psi}}}{(1+a) \mathrm{e}^{-\hat{\psi}}+(1-a) \mathrm{e}^{\hat{\psi}}}\right]
$$

In terms of the circular velocity $v_{c}$ the specific angular momentum can be written as

$$
L=\frac{\rho e^{-\psi} v_{c}}{\sqrt{1-v_{c}^{2}}}+\tilde{e} A .
$$

To analyze the stability of circular orbits on the equatorial plane we use an extension of Rayleigh criteria of stability of a fluid at rest in a gravitational field [68 70]. The motion equation (90) can be cast as a balance equation

$$
\frac{g^{\rho \rho} g_{t t, \rho} E^{2}}{2 g_{t t}^{2}}+\tilde{e} g^{\rho \rho} A_{, \rho} \omega u^{0}=-\frac{g^{\rho \rho} g_{\varphi \varphi, \rho}(L-\tilde{e} A)^{2}}{2 g_{\varphi \varphi}^{2}}
$$

where the term first on the left-hand side represents the gravitational force $F_{g}$, the term second the the Lorentz force $F_{L}$, and the term on the right-hand side the centrifugal force $F_{c}(\rho)=F(\rho, L(\rho))$ acting on the particle. So we have a balance between the total force $F(\rho)=F_{g}+F_{L}$ and the centrifugal force. We now consider the particle to be initially in a circular orbit with radius $\rho=\rho_{0}$ and we slightly displace it to a higher orbit $\rho>\rho_{0}$. The angular momentum of particle remains equal to its initial value $L_{0}=L\left(\rho_{0}\right)$ which implies that the centrifugal force in its new position is $F_{c}\left(\rho, L_{0}\right)$. In order that the particle returns to it initial position must be met that $F(\rho)>F_{c}\left(\rho, L_{0}\right)$, but according to the balance equation (103) $F(\rho)=F_{c}(\rho, L)$ so that $F_{c}(\rho, L)>F_{c}\left(\rho, L_{0}\right)$, and hence $(L-\tilde{e} A)^{2}>\left(L_{0}-\tilde{e} A\right)^{2}$. Using the expresion for $L$ (97) and defining la function $h=g_{\varphi \varphi} \omega u^{0}$, follows that $h(\rho)^{2}>h\left(\rho_{0}\right)^{2}$. Note that the quantity $h$ has the same form that the specific angular momentum in the vacuum. By doing a Taylor expansion of $h^{2}(\rho)$ around $\rho=\rho_{0}$ one finds that the condition of stability for a circular orbit is

$$
h h_{, \rho}>0
$$

or, in other words,

$$
h_{, \rho}^{2}>0
$$

In order to study the behavior of these physical quantities for the previous solutions again we perform a graphical analysis of them for the zeroth and second terms of the series in the three families of solutions considered. In all cases we take $\tilde{e}=1$. We first analyze the magnetized Weyl solutions in spherical coordinates. In this case $A_{, \rho}=0$, so that the velocity is given by expression (100). In Figure 5 we show the circular velocity curves $v_{c}^{2}$ and $h^{2}$ for test particles with $c_{0}=0.5$ and $c_{2}=1$ (figures $\underline{5}(a)$ and $\underline{5}(b)$ ), and $c_{0}=0$ and $c_{2}=-0.5$ 
(figures $5(c)$ and $5(d)$ ), for values of magnetic field parameter $b=0$ (dashed curves), 1, 2, 3 (top curves), as functions of $\rho$. In first case we see that the tangential velocity increase initially from certain $\rho=\rho_{0}$, reaches a maximum and then falls to zero, and is always a quantity less than the velocity of light. We also observer that inclusion of magnetic field make these orbits less relativistic. Meantime we see that the quantity $h^{2}$ always is a increasing function of $\rho$ that corresponds to stable circular orbits. In the second case we find that $v_{c}^{2}$ increases from zero at infinity to 1 (the velocity of light) at circular photon orbit $\rho=\rho_{p h}$, and that $h^{2}$ always is a decreasing monotonous function of $\rho$ which means that this orbits are instable against radial perturbation.

We now analyze the magnetized Erez-Rosen solutions. In this case also $A_{,}=0$. So in figure 6 we show the circular velocity curves $v_{c}^{2}$ and $h^{2}$ for test particles with $c_{0}=0.5$ and $c_{2}=1$ (figures $6(a)$ and $6(b)$ ), and $c_{0}=0$ and $c_{2}=-0.5$ (figures $6(c)$ and $6(d)$ ), for values of magnetic field parameter $b=0$ (dashed curves), 0.5, 1, 2 (top curves), as functions of $\rho$. In first case we see that the tangential velocity increase initially, reaches a maximum and then falls to zero, being always a quantity less than the velocity of light. We also observer that inclusion of magnetic field make these orbits less relativistic. The quantity $h^{2}$ always is a increasing function of $\rho$ that corresponds to stable circular orbits. In the second case we find that $v_{c}^{2}$ also increase initially but not from zero and that only the central regions are stable.

In Figures 7 and 8 we show for magnetized first order Morgan-Morgan fields the circular velocity curves $v_{c}^{2}$ and $h^{2}$ for test particles with $c_{0}=0.5, c_{2}=1$ (figures 7) and with $c_{0}=0, c_{2}=-1$ (figures 8) for values of magnetic field parameter $b=0,1$, and 1.4. We find inside of the disk $A_{, \rho} \neq 0$ so that in this case we have two values for the angular velocity $\omega_{ \pm}$, which corresponds to the direct and retrograde motions of particles. Figures on the left side correspond to the inside of the disk and to the direct motion of particles. For the retrograde motion of the particles we obtain a similar behavior. For all values of parameters we find regions where circular orbits are possible, that is, regions where the velocity of particles is always a quantity less than the velocity of light. We also observer that inclusion of magnetic field can makes these orbits less relativistic. We find strong change in the slope of $h^{2}$ at certain values of $\rho$ which means that there is a strong instability there, also regions with negative slope where the orbits are unstable, but always we find regions where $h_{+}^{2}$ is a increasing monotonous function of $\rho$ that corresponds to stable circular orbits. We also observe that the increase of magnetic field can make stable these orbits against radial perturbation.

In order to compare the behavior of these quantities with other known magnetized solutions we now analyze a Kerr type solution or magnetic dipole solution 74 . In this case also we have two values for the angular velocity, $\omega_{ \pm}$. For the direct motion of the particles, in figure 9 we present $9(a)$ the circular velocity curves $v_{c+}^{2}$ for $b=0$ (dashed curve), 0.5 (curve with points and lines), 1.5, 2, 2.5, and 3 (bottom curve), and $h_{+}^{2}$ for $9(b) b=0$ (dashed curve), 0.5, 9 (c) $b=1.5$ (dashed curve), 2, 2.5, and 3 (bottom curve), as functions of $\rho$. We see that in absence of magnetic field $b=0$ the circular velocity increases from zero at infinity to 1 (the velocity of light) at circular photon orbit $\rho=\rho_{p h}$, but as we increase the magnetic field it reaches a maximum and then falls to zero, being always less than the velocity of light. We also see that inclusion of magnetic field make these orbits less relativistic. Regarding the function $h^{2}$ we find for $b=0$ and $b=0.5$ (or weak fields) strong change in the slope of $h_{+}^{2}$ at certain values of $\rho$ which means that there is a strong instability there, also regions with negative slope where the orbits are unstable, but after certain values of $\rho$ we find that $h_{+}^{2}$ is a increasing monotonous function of $\rho$ that corresponds to stable circular orbits. We also observe that the increase of magnetic field can make stable these orbits against radial perturbation. For the retrograde motion of the particles we obtain a similar behavior.

\section{DISCUSSION}

Using the well-known Ernst's method were constructed three families of general magnetostatic axisymmetric exact solutions of Einstein-Maxwell equations in spherical coordinates, prolate, oblates, and also in generalized spheroidal coordinates which is a generalization of the previous systems. Except the zero order, the constructed solutions are asymptotically flat but with total mass zero. Hence, all discussed solutions with magnetic field are either singular, not asymptotically flat or massless. In the three cases considered we show explicitly the solutions corresponding to the zeroth and second terms of the series of the solutions, among them a magnetized Erez-Rosen metric and a magnetized Morgan-Morgan metric, which was interpreted as the exterior gravitational field of a finite dislike source immersed in a magnetic field, and we analyze the material properties of the disk such as the surface energy density and the azimuthal pressure. From them we also study relativistic models of thin disks of infinite extension. We find always values of parameters for which all the energy conditions are satisfied.

We also analyzed the electrogeodesic equatorial circular motion of test particles for the sum of the first and second terms of series of solutions and we also discuss their stability. For all values of parameters we find regions where circular orbits are possible, that is, regions where the circular velocity of particles is always a quantity less than the velocity of light. We also observer that inclusion of magnetic field can makes these orbits less relativistic. We find strong change in the slope of the function $h^{2}$ at certain values of the radial coordinate $\rho$ which means that there is a strong instability there, also regions with negative slope where the circular orbits are unstable, but always we find 
values of parameter for which $h^{2}$ is a increasing monotonous function of $\rho$ that corresponds to stable orbits. We also observe that the increase of magnetic field can make stable these orbits against radial perturbation. For magnetized Morgan-Morgan fields was found that inside of disk the presence of magnetic field provides the possibility of to find relativist charged particles moving in both direct and retrograde direction.

Finally in order to compare the behavior of these physical quantities with other known magnetized solutions, we also analyze the electrogeodesic motion of test particles and their stability for a Kerr-type solution (magnetic dipole solution). We found for some value of the parameter a similar behavior to the previous cases.

[1] A.P. Lobanov, A\&A 330, 79 (1998).

[2] F.A. Aharonian, MNRAS 332, 215 (2002).

[3] A.F. Zakharov et al, MNRAS 342, 1325 (2003).

[4] J.S. Greaves et al, Nature 404, 732 (2000).

[5] Y.T. Liu, S.L. Shapiro and B.C. Stephens, Phys. Rev. D 76, 084017 (2007).

[6] S. Akiyama et al, ApJ 584, 954 (2003).

[7] W.H.T. Vlemmings, P.J. Diamond and H. Imai, Nature 440, 58 (2006).

[8] D.L. Meier, S. Koide and Y. Uchida, Science 291, 84 (2001).

[9] G.V. Ustyugova et al, ApJ 516, 221 (1999).

[10] R. Krasnopolsky, Z.Y. Li and R.D. Blandford, ApJ 595, 631 (2003).

[11] D.N. Aguilera, J.A. Pons and J.A. Miralles, ApJ 673, L167 (2008).

[12] P. Hennebelle and S. Fromang, A\&A 477, 9 (2008).

[13] Z. Medin and D. Lai, MNRAS 382, 1833 (2007).

[14] X.D. Li and E.P.J. van den Heuvel, ApJ 513, L45 (1999).

[15] G.G. Pavlov and V.G. Bezchastnov, ApJ 635, L61 (2005).

[16] A.I. Ibrahim, J.H. Swank and W. Parke, ApJ 584, L17 (2003).

[17] K. Schwarzschild, Sitzungsberichte der Kniglich Preussis- chen Akademie der Wissenschaften 1, 189 (1916).

[18] K. Schwarzschild, Sitzungsberichte der Kniglich Preussis- chen Akademie der Wissenschaften 1, 424 (1916).

[19] R.P. Kerr, Phys. Rev. Lett. 11, 237 (1963).

[20] E.T. Newman et al, J. Math. Phys. 6, 918 (1965).

[21] H. Reissner, Ann. Phys. Berlin 50, 106 (1916).

[22] G. Nordstr m, Verhandl. Koninkl. Ned. Akad. Weten-oschap. 20, 1231 (1918).

[23] J. Polanco, P. Letelier, and M. Ujevic, Phys. Rev. D 78, 024026 (2008).

[24] W. A. Bonnor and A. Sackfield, Commun. Math. Phys. 8, 338 (1968).

[25] T. Morgan and L. Morgan, Phys. Rev. 183, 1097 (1969).

[26] L. Morgan and T. Morgan, Phys. Rev. D 2, 2756 (1970).

[27] G. A. González and P. S. Letelier, Class. Quantum Grav. 16, 479 (1999).

[28] D. Lynden-Bell and S. Pineault, Mon. Not. R. Astron. Soc. 185, 679 (1978).

[29] A. Chamorro, R. Gregory, and J. M. Stewart, Proc. R. Soc. London A413, 251 (1987).

[30] P.S. Letelier and S. R. Oliveira, J. Math. Phys. 28, 165 (1987).

[31] J. P. S. Lemos, Class. Quantum Grav. 6, 1219 (1989).

[32] J. P. S. Lemos and P. S. Letelier, Class. Quantum Grav. 10, L75 (1993).

[33] J. Bičák, D. Lynden-Bell, and J. Katz, Phys. Rev. D 47, 4334 (1993).

[34] J. Bičák, D. Lynden-Bell, and C. Pichon, Mon. Not. R. Astron. Soc. 265, 126 (1993).

[35] G.A. González and O. A. Espitia, Phys. Rev. D 68, 104028 (2003).

[36] J. Bičák and T. Ledvinka, Phys. Rev. Lett. 71, 1669 (1993).

[37] G. A. González and P. S. Letelier, Phys. Rev. D 62, 064025 (2000).

[38] T. Ledvinka, J. Bičák, and M. Z̆ofka, in Proceeding of 8th Marcel-Grossmann Meeting in General Relativity, edited by T. Piran (World Scientific, Singapore, 1999)

[39] G. García-Reyes and G. A. González, Brazilian Journal of Physics 37, no. 3B, 1094 (2007).

[40] P. S. Letelier, Phys. Rev. D 60, 104042 (1999).

[41] J. Katz, J. Bičák, and D. Lynden-Bell, Class. Quantum Grav. 16, 4023 (1999).

[42] G. García R. and G. A. González, Phys. Rev. D 69, 124002 (2004).

[43] D. Vogt and P. S. Letelier, Phys. Rev. D 70, 064003 (2004).

[44] G. García-Reyes and G. A. González, Class. Quantum Grav. 21, 4845 (2004).

[45] G. García-Reyes and G. A. González, Phys. Rev. D 70, 104005 (2004).

[46] J. L. Synge, Relativity: The General Theory. (North-Holland, Amsterdam, 1966).

[47] G. Neugebauer and R. Meinel, Phys. Rev. Lett. 75, 3046 (1995).

[48] C. Klein, Class. Quantum Grav. 14, 2267 (1997).

[49] C. Klein and O. Richter, Phys. Rev. Lett. 83, 2884 (1999).

[50] C. Klein, Phys. Rev. D 63, 064033 (2001).

[51] J. Frauendiener and C. Klein, Phys. Rev. D 63, 084025 (2001). 
[52] C. Klein, Phys. Rev. D 65, 084029 (2002).

[53] C. Klein, Phys. Rev. D 68, 027501 (2003).

[54] C. Klein, Ann. Phys. (Leipzig) 12, 599 (2003).

[55] D. Vokrouhlicky and V. Karas, Astron. Astrophys. 243, 165 (1991).

[56] S. Sengupta, Int. J. Mod. Phys. D 6, 591 (1997).

[57] F. de Felice and F. Sorge, Class. Quantum Grav. 20, 469 (2003).

[58] J. Kovář, O. Kopáček, V. Karas and Z Stuchlík, Class. Quantum Grav. 27, 135006 (2010).

[59] J. Frank, A. King and D. Raine, Accretion Power in Astrophysics (Cambridge: Cambridge University Press, 1992).

[60] G. Belvedere, Proc. European Physical Society Study Conference, Accretion Disks and Magnetic Fields in Astrophysics (Dordrecht: Kluwer, 1998).

[61] A. N. Aliev and N. Ozdemir, Mon. Not. Roy. Astron. Soc. 336, 241 (2002).

[62] J. Braithwaite and H. C. Spruit, Nature 431, 819 (2004).

[63] V. C. Rubin, J. A. Graham and J. D. P Kenney. Ap. J. 394, L9, (1992).

[64] H. Rix, M. Franx, D. Fisher and G. Illingworth. Ap. J. 400, L5, (1992).

[65] F. Bertola et al. Ap. J. 458, L67 (1996).

[66] C. Struck, Phys. Rep. 321, 1 (1999).

[67] R. Ciri, D. Bettoni, and G. Galletta, Nature 375, 661 (1995).

[68] Lord Rayleigh, 1917, Proc. R. Soc. London A, 93, 148

[69] L. D. Landau and E.M. Lifshitz, Fluid Mechanics(Addison-Wesley, Reading, MA, 1989).

[70] P. S. Letelier, Phys. Rev. D 68, 104002 (2003).

[71] F. J. Ernst. Phys. Rev. 167, 1175 (1968).

[72] F. J. Ernst. Phys. Rev. 168, 1415 (1968).

[73] D. Kramer, H. Stephani, E. Herlt, and M. McCallum, Exact Solutions of Einsteins's Field Equations (Cambridge University Press, Cambridge, England, 1980).

[74] W. B. Bonnor, Z. Phys. 190, 444 (1966).

[75] Bateman, H. Higher Transcendental Functions. McGraw Hill, New York, vol. 2

[76] Arfken, G. Mathematical Methods for Physicists. Academic Press.

[77] S. M. Scott and P. Szekeres, Gen. Rel. Grav., 18, 557 (1986).

[78] S. M. Scott and P. Szekeres, Gen. Rel. Grav., 18, 571 (1986).

[79] J. M. Bardeen, B. Carter, S. W. Hawking, Commun. Math. Phys., 31, 161 (1973).

[80] H. Quevedo, Phys. Rev. D 39, No. 10, 2904 (1989).

[81] Chazy J 1924 Bull. Soc. Math., France 5217.

[82] Curzon H E J 1924 Proc. London Math. Soc. 23477.

[83] S. Chandrasekhar, The Mathematical Theory of Black holes (Oxford Publications, New York, 1992 ), p. 623.

[84] D. M. Zipoy. J. Math. Phys. 7, 1137 (1966).

[85] B. H. Voorhees. Phys. Rev. D 2, 2119 (1970).

[86] K. Schwarzschild. Sitzungsber. Preuss. Akad. Wiss., 189 (1916).

[87] G. Erez and N. Rosen, Bull. Res. Counc. Isr. 8F, 47 (1959).

[88] W. A. Bonnor and A. Sackfield, Comm. Math. Phys. 8, 338 (1968).

[89] G. A. González and J. F. Ramos, Rev. Col. Fís. 33, No.2, 118 (2001).

[90] A. Papapetrou and A. Hamouni, Ann. Inst. Henri Poincaré 9, 179 (1968)

[91] A. Lichnerowicz, C.R. Acad. Sci. 273, 528 (1971)

[92] A. H. Taub, J. Math. Phys. 21, 1423 (1980)

[93] E. Israel, Nuovo Cimento 44B, 1 (1966)

[94] E. Israel, Nuovo Cimento 48B, 463 (1967)

[95] G. G. Kuzmin 1956, Astron. Zh., 33, 27 (1956)

[96] A. Toomre, Ap. J., 138, 385 (1962) 


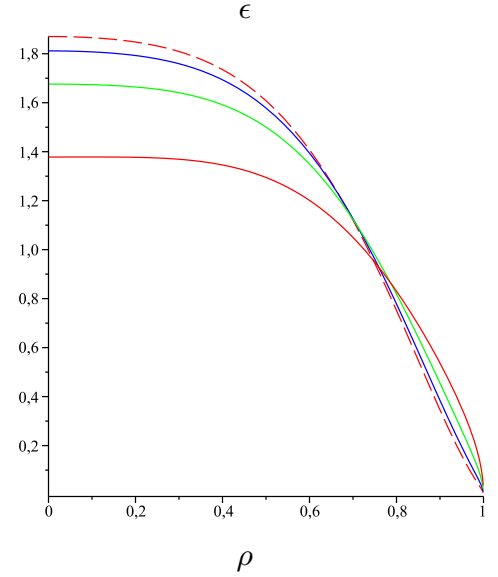

$(a)$

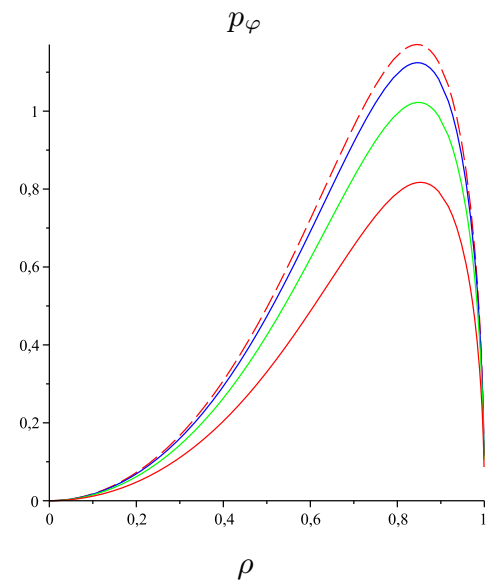

$(b)$

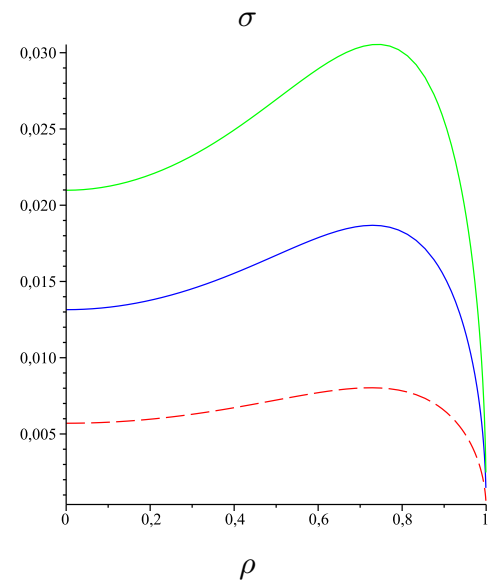

$(c)$

FIG. 1. (a) The surface energy density $\epsilon$ and $(b)$ the azimuthal pressure $p_{\varphi}$ for magnetized first order Morgan-Morgan finite disks with $c_{0}=c_{2}=0.4$ and for values of magnetic field parameter $b=0$ (dashed curves), 0.5, 1 , and 2 (bottom curves), as functions of $\rho$. (c) The surface electric charge density $\sigma$ (electrostatic case) for $p=0.2$ (dashed curve), 0.5 , and 1 (top curve) and the same values of $c_{0}$ and $c_{2}$.

$\epsilon$

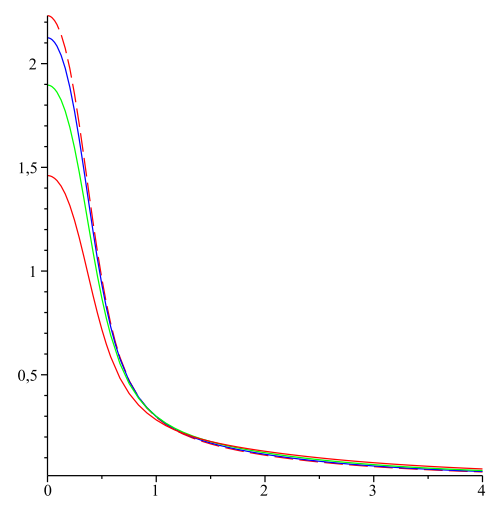

$(a)$ $p_{\varphi}$

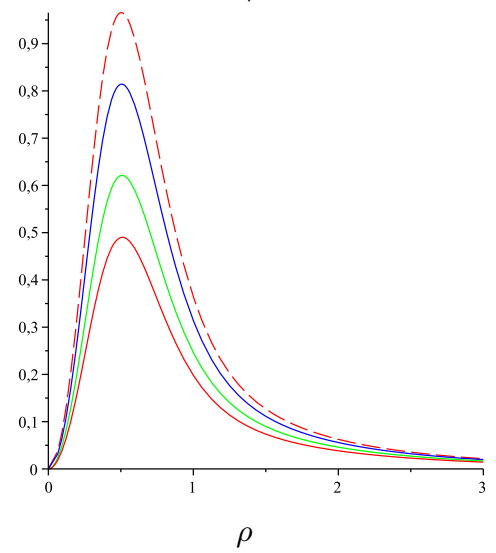

(b)

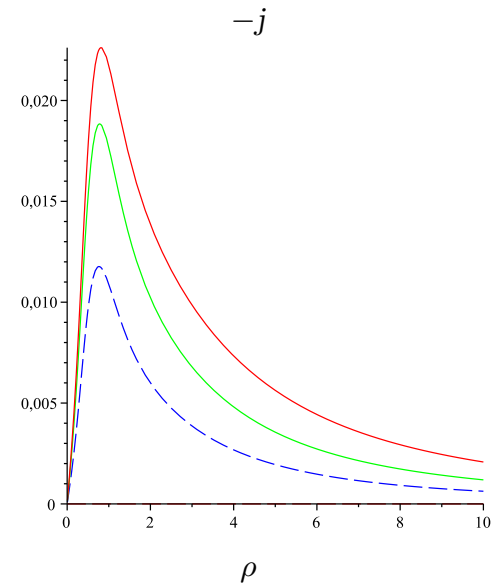

(c)

FIG. 2. (a) The surface energy density $\epsilon$ and $(b)$ the azimuthal pressure $p_{\varphi}$ for magnetized weyl type infinite disks in spherical coordinates and second order with $z_{0}=1, c_{0}=1, c_{2}=0.5$ and for values of magnetic field parameter $b=0$ (dashed curves), $0.5,1$, and 2 (bottom curves), as functions of $\rho$. (c) The azimuthal current density $j$ for $b=0.5$ (dashed curve), 1 , and 2 (top curve) and the same values of $z_{0}, c_{0}$ and $c_{2}$. 
$\epsilon$

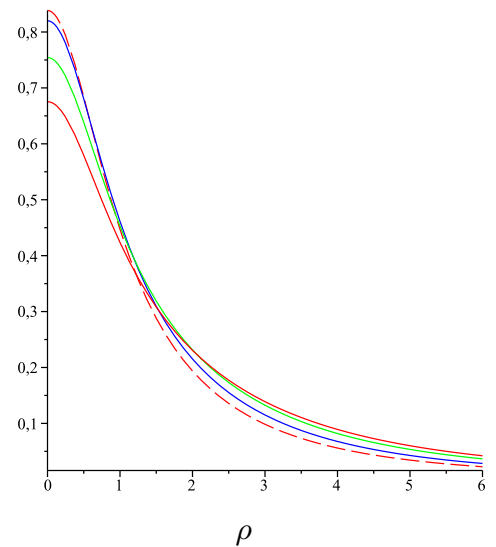

$(a)$ $p_{\varphi}$

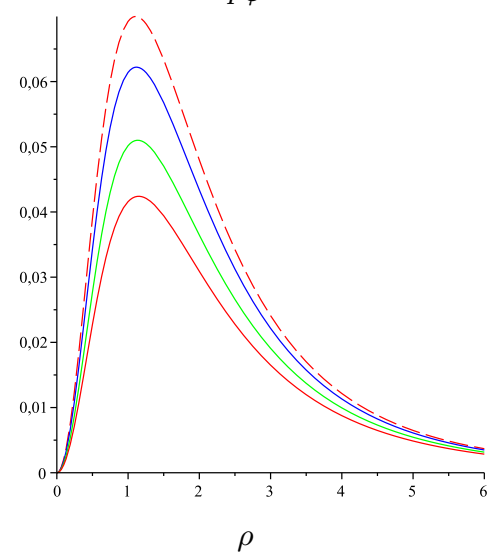

(b)

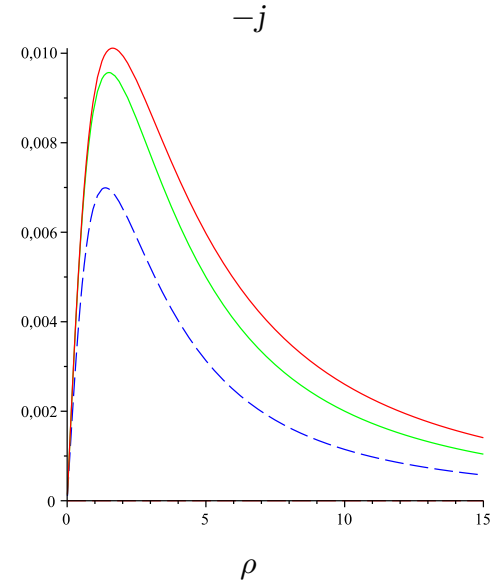

(c)

FIG. 3. (a) The surface energy density $\epsilon$ and $(b)$ the azimuthal pressure $p_{\varphi}$ for magnetized Erez-Rosen type infinite disks with $z_{0}=2, c_{0}=c_{2}=1$ and for values of magnetic field parameter $b=0$ (dashed curves), 1, 2, and 3 (bottom curves), as functions of $\rho$. (c) The azimuthal current density $j$ for $b=1$ (dashed curve), 2, and 3 (top curve) and the same values of $z_{0}, c_{0}$ and $c_{2}$.

$\epsilon$

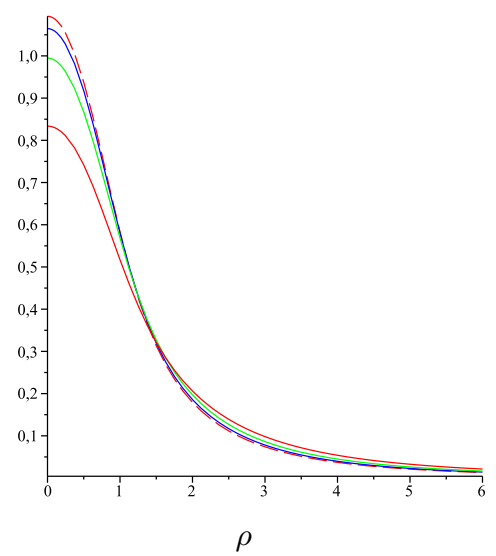

$(a)$ $p_{\varphi}$

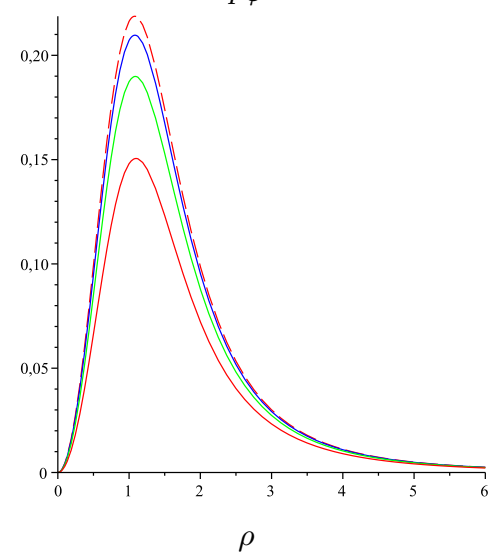

(b)

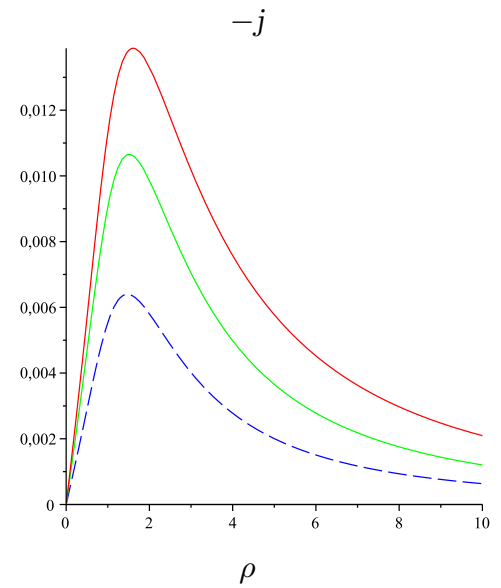

(c)

FIG. 4. (a) The surface energy density $\epsilon$ and $(b)$ the azimuthal pressure $p_{\varphi}$ for magnetized first order Morgan-Morgan type infinite disks with $z_{0}=c_{0}=c_{2}=1$ and for values of magnetic field parameter $b=0$ (dashed curves), 0.5, 1, and 2 (bottom curves), as functions of $\rho$. (c) The azimuthal current density $j$ for $b=0.5$ (dashed curve), 1 , and 2 (top curve) and the same values of $z_{0}, c_{0}$ and $c_{2}$. 
$v_{c}^{2}$

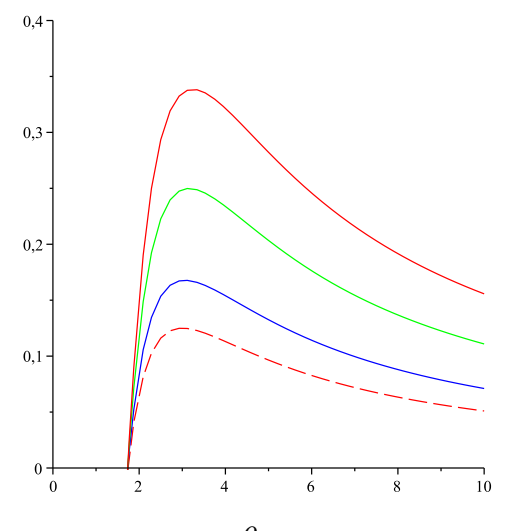

$(a)$

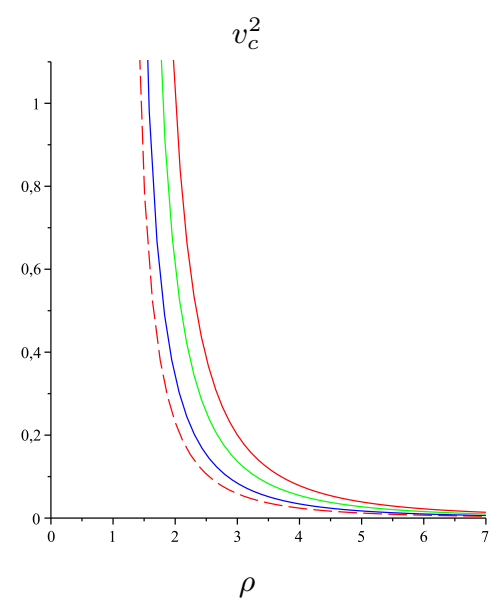

(c) $h^{2}$

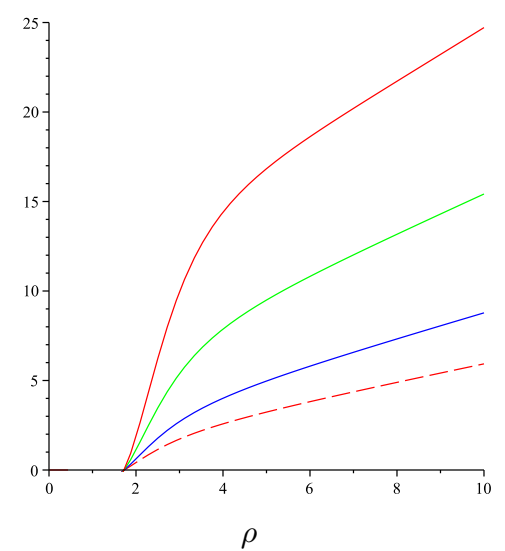

$(b)$

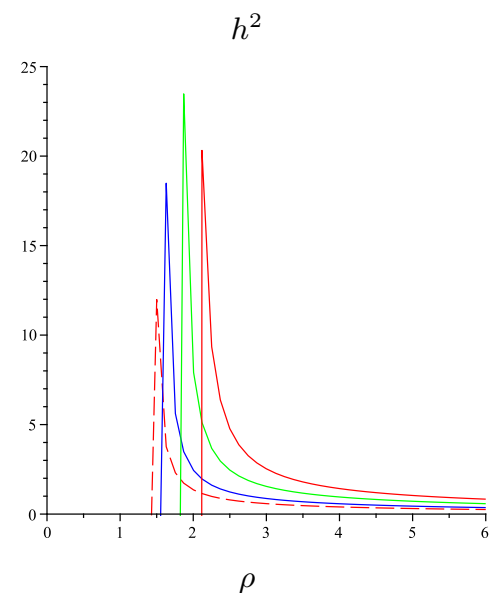

$(d)$

FIG. 5. For magnetized weyl fields in spherical coordinates and second order we plot, as functions of $\rho$, the circular velocity $v_{c}^{2}$ and $h^{2}$ for test particles with $c_{0}=0.5$ and $c_{2}=1$ (figures $(a) \mathrm{y}(b)$ ), and $c_{0}=0$ and $c_{2}=-1$ (figures $(c)$ y $(d)$ ), for values of magnetic field parameter $b=0$ (dashed curves), 1, 2, 3 (top curves). 

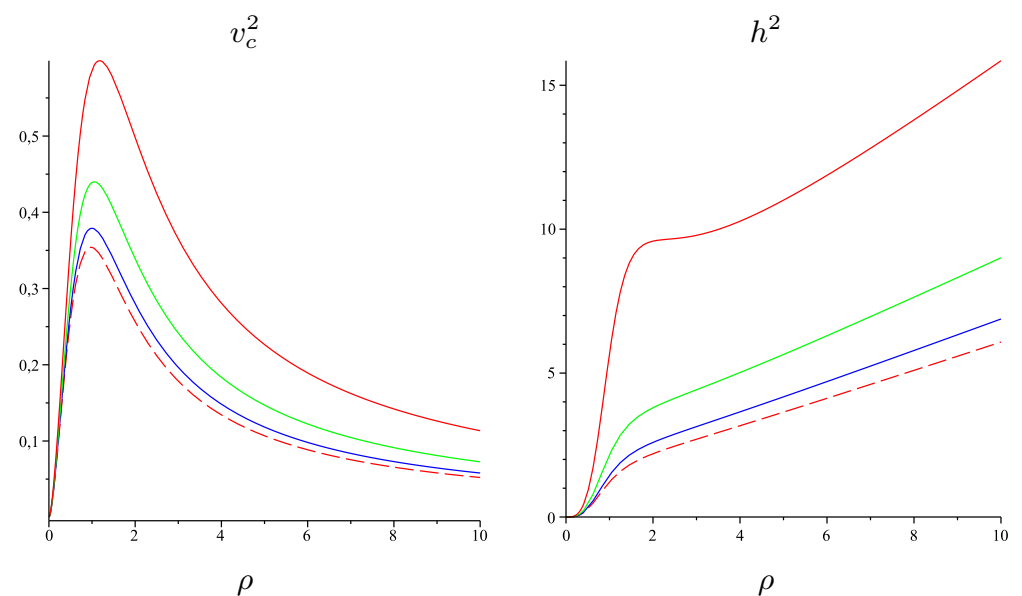

$(a)$

$(b)$

$v_{c}^{2}$

$h^{2}$

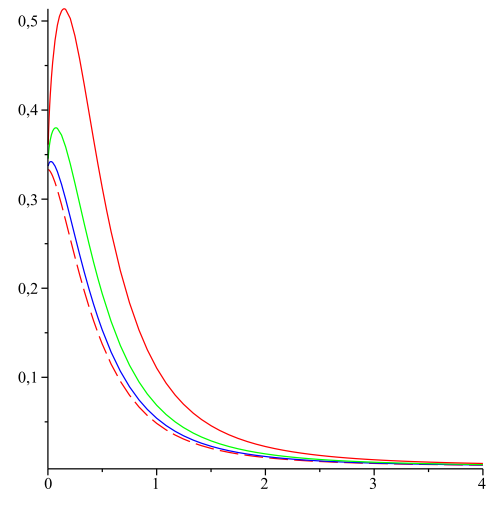

$(c)$

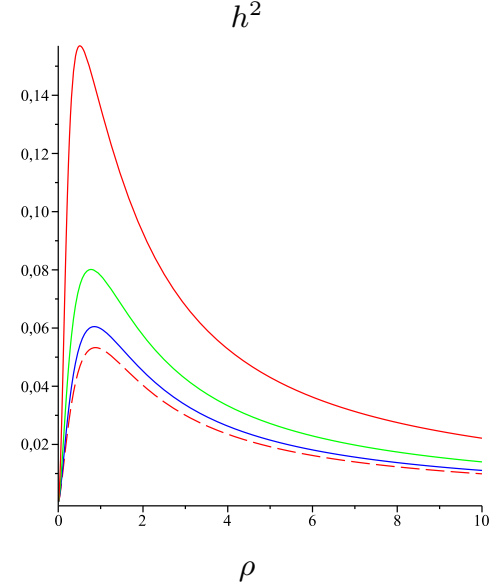

(d)

FIG. 6. For magnetized Erez-Rosen fields we plot, as functions of $\rho$, the circular velocity $v_{c}^{2}$ and $h^{2}$ for test particles with $c_{0}=0.5$ and $c_{2}=1$ (figures $(a)$ y $(b)$ ), and $c_{0}=0$ and $c_{2}=-0.5$ (figures $(c)$ y $(d)$ ), for values of magnetic field parameter $b=0$ (dashed curves), $0.5,1,2$ (top curves). 


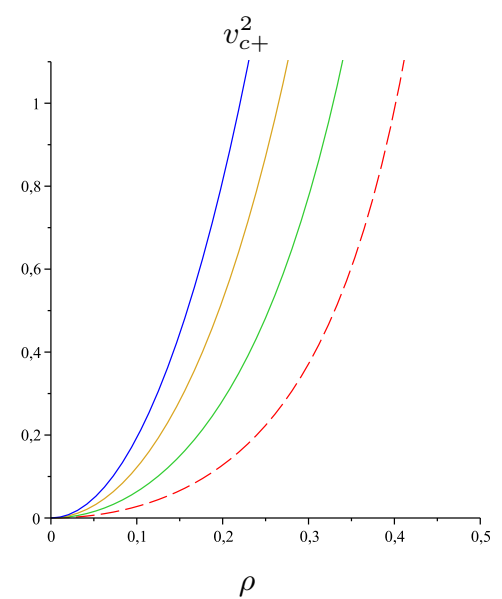

$(a)$

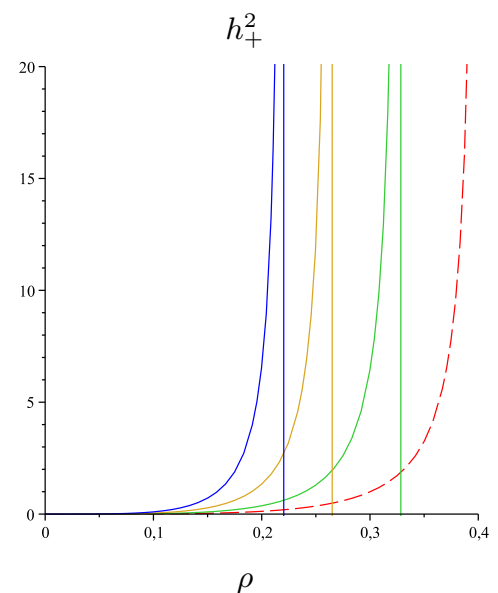

$(c)$

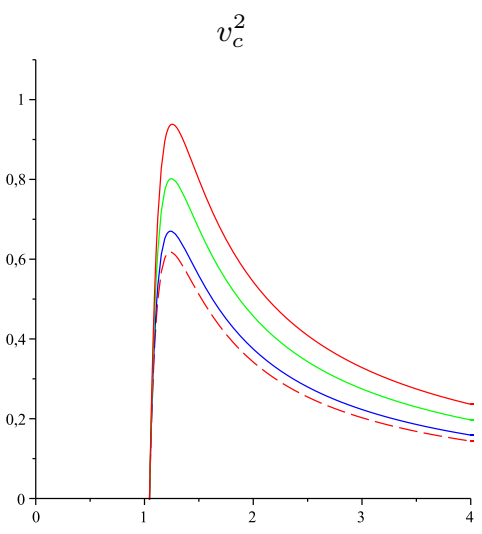

$(b)$

$h^{2}$

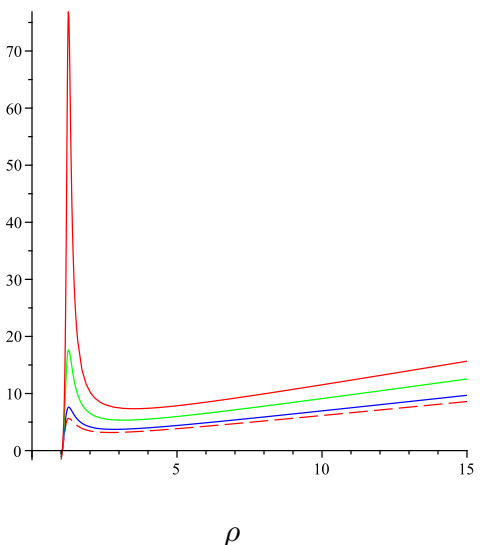

$(d)$

FIG. 7. For magnetized first order Morgan-Morgan fields we plot, as functions of $\rho$, the circular velocity $v_{c}^{2}$ and $h^{2}$ for test particles with $c_{0}=0.5$ and $c_{2}=1$, for values of magnetic field parameter $b=0$ (dashed curves), $0.5,1$, and 1.4 (top curves). Figures on the left side correspond to the inside of the disk and to the motion direct of particles. 


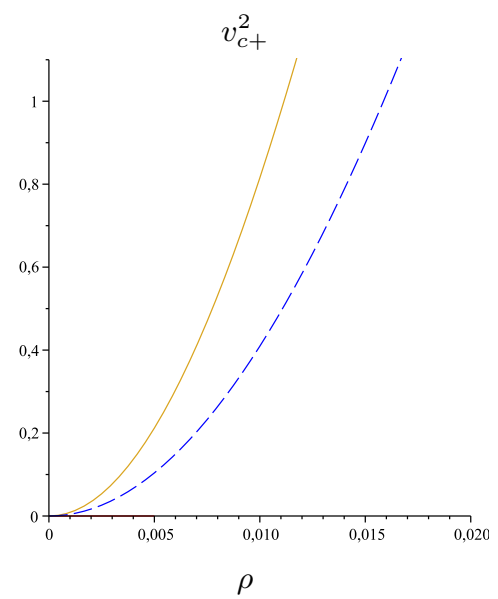

(a)

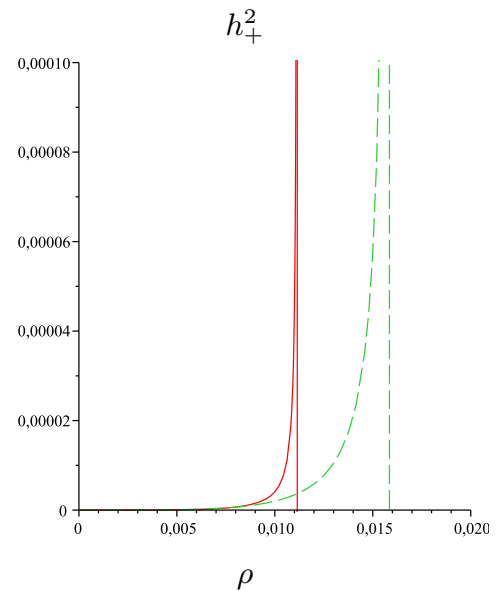

(c)

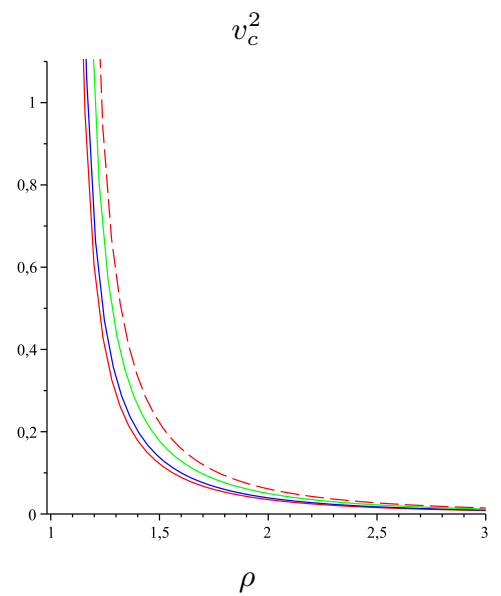

(b)

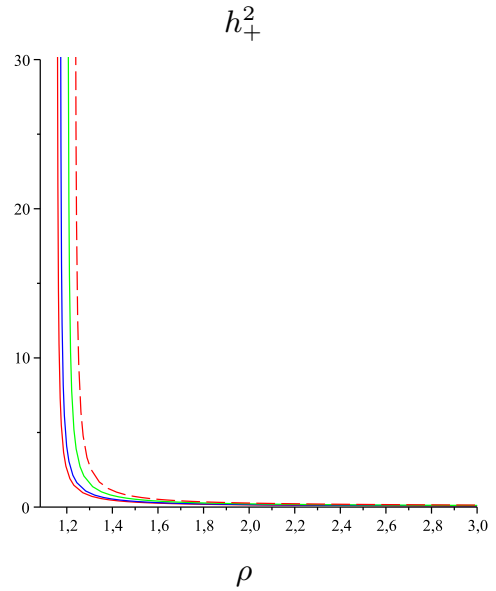

(d)

FIG. 8. Also for magnetized first order Morgan-Morgan fields we plot, as functions of $\rho$, the circular velocity $v_{c}^{2}$ and $h^{2}$ for test particles with $c_{0}=0$ and $c_{2}=-1$, for values of magnetic field parameter $b=0,0.5,1,1.4$ (dashed curves). Again figures on the left side correspond to the interior region of the disk and the motion direct of particles. 


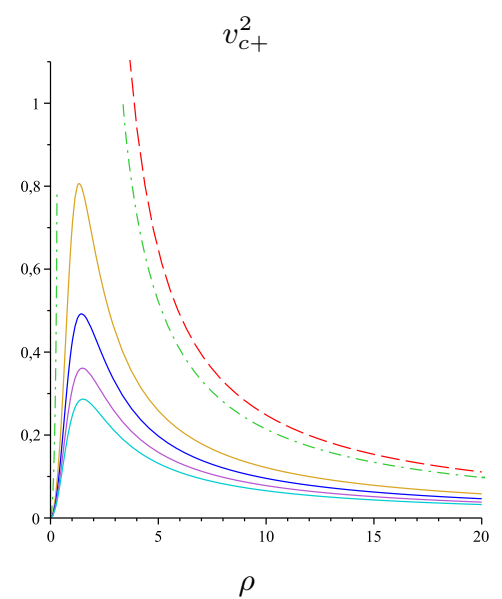

$(a)$

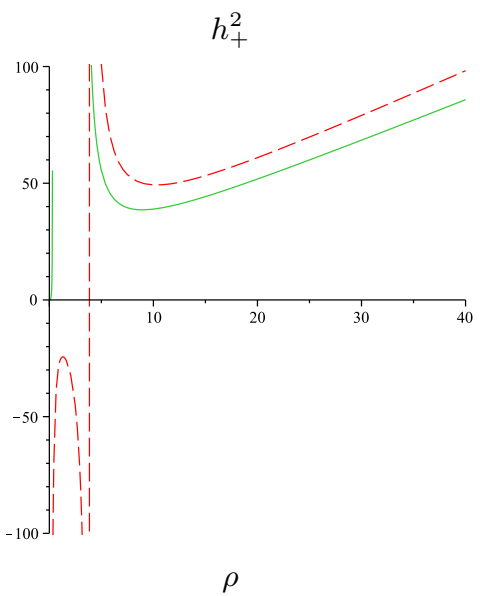

(b)

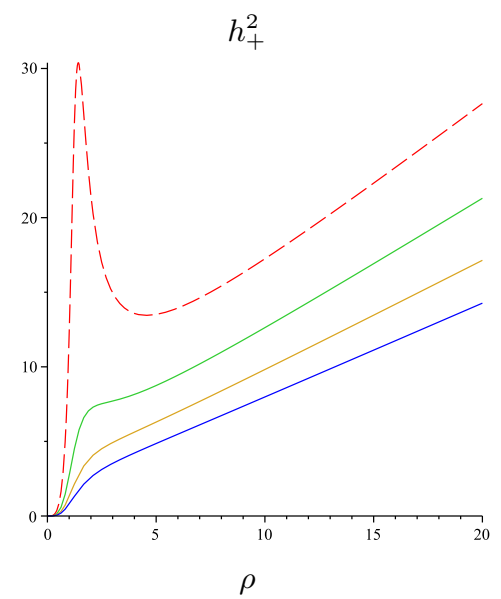

$(c)$

FIG. 9. For a Kerr-type solution (magnetic dipole solution) we plot, as functions of $\rho,(a)$ the circular velocity $v_{c+}^{2}$ for test particles with $b=0$ (dashed curve), 0.5 (curve with points and lines), 1.5, 2, 2.5, and 3 (bottom curve), and $h_{+}^{2}$ for $(b) b=0$ (dashed curve), 0.5, (c) $b=1.5$ (dashed curve), 2, 2.5, and 3 (bottom curve). 